\title{
Calculation of the average travel distance in a low-level picker-to-part system considering any distribution function within the aisles
}

\author{
DR.-ING. VOLKER SADOWSKY \\ Prof. Dr. MichaEl TEN HOMPEL \\ CHAIR OF MATERIAL HANDLING AND WAREHOUSING, TU DORTMUND UNIVERSITY
}

\begin{abstract}
The estimation of the average travel distance in a low-level picker-to-part order picking system can be done by analytical methods in most cases. Often a uniform distribution of the access frequency over all bin locations is assumed in the storage system. This only applies if the bin location assignment is done randomly. If the access frequency of the articles is considered in the bin location assignment to reduce the average total travel distance of the picker, the access frequency over the bin locations of one aisle can be approximated by an exponential density function or any similar density function. All known calculation methods assume that the average number of orderlines per order is greater than the number of aisles of the storage system. In case of small orders this assumption is often invalid. This paper shows a new approach for calculating the average total travel distance taking into account that the average number of orderlines per order is lower than the total number of aisles in the storage system and the access frequency over the bin locations of an aisle can be approximated by any density function.

\section{Zusammenfassung}

Die Ermittlung der Kommissionierleistung in Person-zur-Ware-Systemen kann in vielen Fällen durch analytische Methoden bestimmt werden. Dabei wird häufig eine Gleichverteilung der Zugriffshäufigkeit auf alle Lagerplätze vorausgesetzt. Dies ist aber nur dann gewährleistet, wenn die Zuordnung der Artikel zu den Lagerplätzen zufällig erfolgt. Wird die Zugriffshäufigkeit der Artikel bei der Lagerplatzzuordnung berücksichtigt, um z.B. die mittlere Wegstrecke des Kommissionierers zu reduzieren, kann die Zugriffshäufigkeit auf die Lagerplätze einer Gasse durch die Dichtefunktion der Exponentialverteilung oder vergleichbaren Verteilungsfunktionen approximiert werden. Alle für diesen Fall bekannten Berechnungsmethoden setzen voraus, dass die Anzahl Positionen, die im Mittel pro Auftrag gepickt werden, größer ist als die Gesamtanzahl der Gassen des Lagers. Diese Voraussetzung ist allerdings bei kleinen Aufträgen häufig nicht gegeben. Im Rahmen dieses Artikels wird ein analytisches Verfahren vorgestellt, dass keine Restriktionen bezüglich der Positionsanzahl und Gassenanzahl aufweist und beliebige Dichtefunktionen für die Beschreibung der Zugriffshäufigkeit über die Lagerplätze berücksichtigt.
\end{abstract}

\section{Introduction}

Order-picking is a central value-adding process within goods distribution systems. Even in modern logistics facilities, order-picking remains one of the major cost factors due to its high demand for human resources [Tom03]. For this reason, each company strives for the most efficient deployment of its staff.

In low-level picker-to-part order-picking systems, in which the picker physically travels to the location of the Stock-keeping Unit (SKU) to pick an item, a considerable amount of costs can be saved by reducing the total travel distances. This reduces the travel time of the picker and increases the productivity. The personnel expenditure and thus the costs can be reduced. Different pick path routing policies are used for obtaining the shortest path over all individual orders. The policy representing the best solution can be determined by either analytical methods or simulation studies.

The order picking process in low-level picker-to-part systems can be classified into two main pick path routing strategies: Return and Traversal. In the Return Strategy, the picker enters the first aisle from the front, picks the required items, and exits through the front of that same aisle before traveling to the next aisle and entering this and all subsequent aisles from the front. Additionally, a Return Strategy With Repetition describes the case the picker enters and exits the aisle for each item picked (usually due to size or weight). In a Traversal Strategy, the picker enters the first aisle from the front, picks the required items, then exits through the rear of that same aisle, enters the next aisle from the rear and continues this looping process for all subsequent aisles. Using the Return Strategy, the average picking-time is influenced by the density function presenting the access frequencies over 
all bin locations of an aisle. In this paper a 'bin location' is defined as a unique physical location within the storage system where a single SKU is located. The term 'access frequency' is defined as the average number of times a picker has to travel to an individual bin location over a certain period of time.

In previously accepted analytical, non-simulative estimation methods, a uniform distribution of the access frequency over all bin locations is often assumed in the storage system [Gud73, Sch96]. To obtain a uniform density function for the access frequency over all bins of an aisle, a random bin assignment has to be realized. Figure 1 shows exemplary the average access frequencies of the bins.

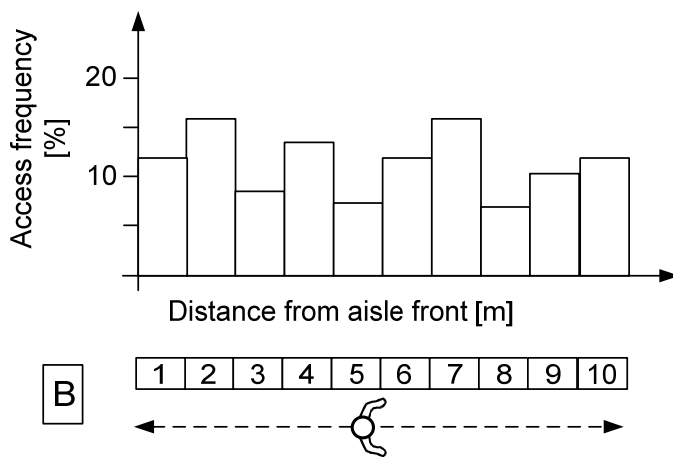

Figure 1: Empirical distribution of access frequencies over the distance from the aisle front (storage bins); random storage bin assignment

The empirical distribution can be modeled approximately by a uniform distribution as shown in Figure 2.

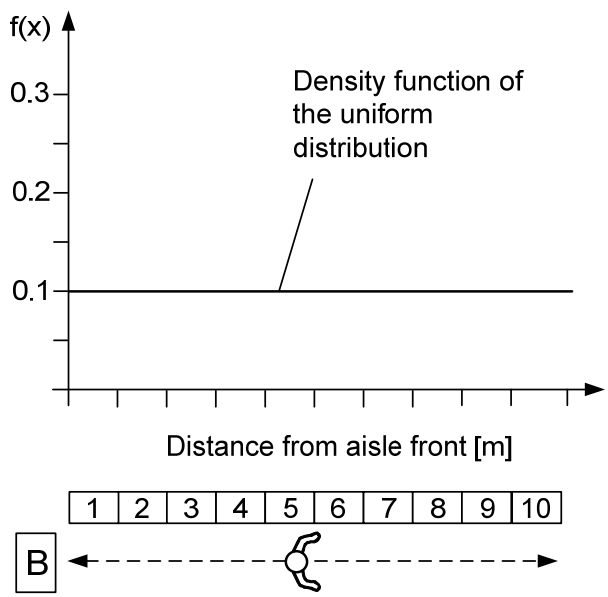

Figure 2: Uniform distributed access frequency over the distance from the aisle front; random storage bin assignment

The following equations show the density and distribution functions of the uniform distribution:

$$
\begin{aligned}
& f(x)= \begin{cases}\frac{1}{b-a} ; & \text { for } a \leq x \leq b \\
0 & \text { else }\end{cases} \\
& F(x)= \begin{cases}\frac{x-a}{b-a} ; & \text { for } a \leq x \leq b \\
0 & \text { else }\end{cases}
\end{aligned}
$$

However, in practice goods are often stored according to their access frequencies (e.g. bin access frequency) to reduce the pickers travel distances. In these cases, the distribution function is not a uniform distribution, but is actually an empirical distribution as shown in Figure 3. 


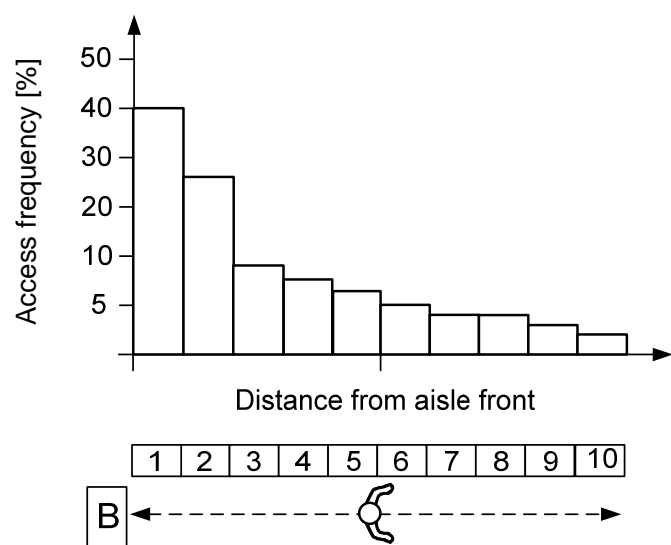

Figure 3: Empirical distribution of access frequencies over the distance from the aisle front, if the access frequency is considered in the bin assignment

This access frequency distribution can be approximated by an exponential density function (Figure.4).

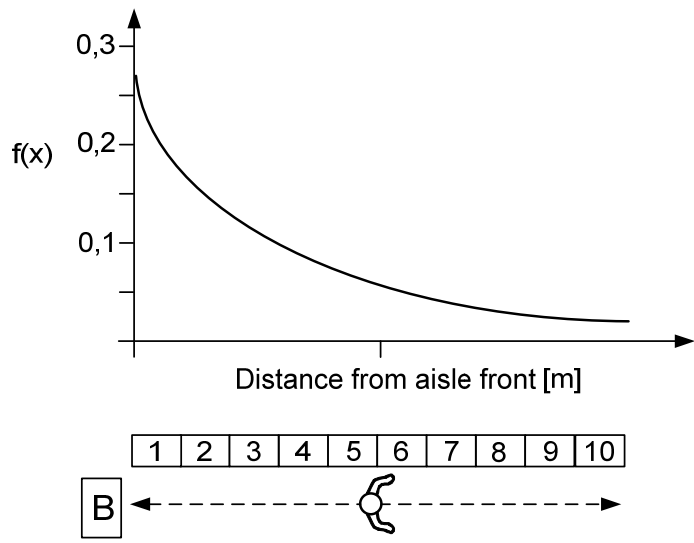

Figure 4: Exponential density function representing the access frequency over the distance from the aisle front, if the access frequency is considered in the bin assignment

The exponential density and distribution functions are defined by the relations

$$
\begin{aligned}
& f(x)= \begin{cases}\lambda \cdot e^{-\lambda \cdot x}, & \text { for } 0 \leq x \leq \infty \\
0, & \text { else }\end{cases} \\
& F(x)= \begin{cases}1-e^{-\lambda \cdot x}, & \text { for } 0 \leq x \leq \infty \\
0, & \text { else }\end{cases}
\end{aligned}
$$

The order picking process in a low-level picker-to-part system is mainly classified into Return and Traversal strategies. The travel time of the Return Strategy is significantly influenced by the density function of the access frequency over all storage bins. The following section describes an estimation method determining the picker's travel time for any distribution function within the aisle. Thus, every bin assignment strategy can be considered. 
Currently no standard notations have been evolved in publications about the estimation of the average travel distance in a picker-to-part system. For a better understanding, the following notations will be used in this paper:

$M=1,2,3, \ldots \quad$ number of SKUs (a unique SKU stored in a single bin location)

$M_{A}=1,2,3, \ldots, M$ number of SKUs per aisle

$N_{A}=1,2,3, \ldots \quad$ number of aisles in the warehouse

$n=1,2,3 \ldots \quad$ average number of orderlines per order

$r=0,1,2,3, \ldots, n \quad$ number of SKUs that have to be picked in a specific aisle

$k=\frac{n}{\chi} \quad$ average number of SKUs that have to be picked in an aisle

$\chi \in[0, \infty] \quad$ average number of aisles approached per order

$s_{n} \in[0, \infty] \quad$ average distance to be covered for picking of $n$ items

$s_{r} \in[0, \infty] \quad$ average distance to be covered in a specific aisle for picking $r$ items

$S_{k} \in[0, \infty] \quad$ average distance in a specific aisle where an average of $k$ items is picked

$s_{B} \in[0, \infty] \quad$ average basic travel distance (constant)

$S_{W} \in[0, \infty] \quad$ average travel distance within an aisle

$s_{A} \in[0, \infty] \quad$ average travel distance across-aisles

$L \in[0, \infty] \quad$ length of an aisle

$L_{C} \in[0, \infty] \quad$ constant length in front of the aisle

$W \in[0, \infty] \quad$ distance between first and last aisle

$W_{A} \in[0, \infty] \quad$ width of an aisle

$W_{C} \in[0, \infty] \quad$ width of a bin location (storage compartment)

$p(r) \in[0,1] \quad$ probability of $r$ items being picked in an aisle

\section{Related work}

The first method for the calculation of travel distances in low-level picker-to-part systems using a Return Strategy was published by Gudehus and Kunder [Gud73] [Kun75]. They assumed that the picker moves onedimensionally within the aisle, that the access frequency is uniformly distributed over all bins, and that the number of items per order is greater than the number of aisles to be picked from [Gud05].

The following three steps are required to calculate the travel distance in a low-level picker-to-part system accordingly: First, the average number of aisles $\chi$ is determined which have to be visited during the picking of $n$ items. Second, the average number of items $k$ picked in an aisle is determined. Third, the distance $s_{k}$ which a picker has to cover in an aisle when picking $k$ items in this aisle is calculated. With this information, the complete within-aisle distance for a single work assignment (e.g. customer order) can be calculated by multiplying $s_{k}$ with the average number of aisles $\chi$.

Ratliff and Rosenthal [Rat83], Goetschalkx and Ratliff [Goe88], Rana [Ran90] and Roodbergen and de Koster [Roo2001] have developed algorithms determining the shortest distance for a single work assignment with a fixed number of items.

Hall's estimation of the mean distances is based on the approaches of Gudehus and Kunder [Ha193]. Based on their results Hall has developed estimation algorithms for the Midpoint Heuristic and the Largest Gap Heuristic, assuming a random distribution of items in the storage system.

Roodbergen and Vis describe a more accurate approach for estimating the travel distance for the Traversal Strategy as well as for the Largest Gap pick path routing strategy [Roo06]. In their approach they also assume that the storage bin assignment is random.

Le Duc and de Koster give an approach for the estimation of the travel distance in a two-block class based storage system [LeD05]. 
Caron has presented an estimation method for the average distance of the Traversal Strategy and the Return Strategy in which the access frequency can be represented by any density function [Car98]. In this particular case, the density function of the Cube-per-Order-Index (COI) bin location assignment strategy is used. The calculations assume a one block layout with an external, central basis.

Hwang transfers Caron's approach to a one block aisle layout with central, internal basis and to any distribution function within the aisles. Additionally, he extends this model by the Midpoint Heuristic [Hwa04].

Caron as well as Hwang have used the Order Statistics laws for the calculation of the average distance in an aisle $s_{k}$ [Car98, Hwa04]. Analog to the method of Gudehus and Kunder, they multiply the average distance within an aisle by the average number of aisles $\chi$ to be approached to determine the within-aisle travel distance $\mathrm{s}_{\mathrm{W}}$. To calculate $\chi$, Hall, Caron and Hwang use the same method as Gudehus and Kunder.

Schulte verifies in his dissertation [Sch96] that the equation for the average number of aisles $\chi$ presented by Gudehus and Kunder only applies if the number of orderlines per order $n$ is higher than the number of existing aisles in the warehouse $N_{A}$ [Gud05].

Based on Combinatorics Theory he develops an equation, which allows the estimation of the average number of visited aisles for those cases that realize a uniformly distributed access frequency within the aisles.

Caron and Hwang use the Order Statistics approach to calculate the average within-aisle distance.

$$
\begin{aligned}
& \begin{aligned}
E\left(X_{(k)}\right) & =\int_{0}^{1} x \cdot \frac{d F^{k}(x)}{d x} d x, \\
\text { with } & k=\frac{n}{\chi}
\end{aligned}
\end{aligned}
$$

Thus $k \in R$. This approach is based on the assumption that ranks are built and that it is ensured that $k \in N$ and $k>0$. Therefore, the equation cannot be formally applied in all cases without the acceptance of roundoff error and the related inaccuracy.

For the reasons mentioned above, none of these approaches offer a general mathematical solution in which the access frequencies within an aisle can be distributed in any way, and the number of orderlines per work assignment is not related to the number of aisles in the storage system.

\section{Calculation of the average travel distances}

To find an analytical solution by means of probability calculation and combinatorics, the following assumptions were made:

- $\quad$ The picking warehouse is a low-level storage system with one or more equally long racks

- All bin locations have the same size (e.g. pallet bin)

- All bin locations contain items of a single SKU

- The access frequencies for all aisles are equal

- The distribution function of the access frequencies versus the distance from bin to aisle front is known

- The distribution function of the access frequencies is the same for all aisles 
The distance $s_{n}$ which is covered in a conventional picking system during the picking of a work assignment with $n$ items, consists of the basic distance $\mathrm{s}_{\mathrm{B}}$, the within-aisles distance $\mathrm{s}_{\mathrm{W}}$ and the across-aisles distance $\mathrm{s}_{\mathrm{A}}$ (Figure 5).

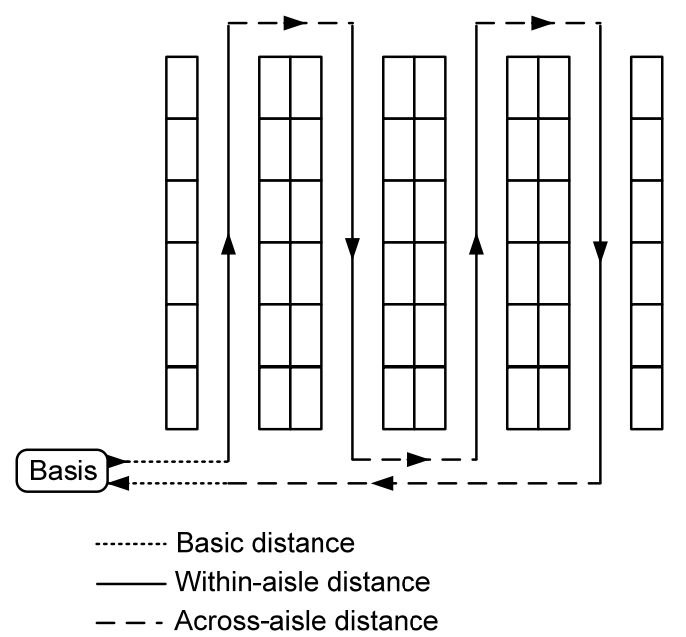

Figure 5: Distances in a low level order picking storage

Thus the average distance to be covered for picking of $n$ items can be described by

$S_{n}=S_{B}+s_{W}+s_{A}$

To estimate the basic distance, the within-aisle distance and the across-aisle distance, certain parameters of the related order picking system have to be known. Figure 6 defines these parameters for a single block layout as well as for a double block layout.
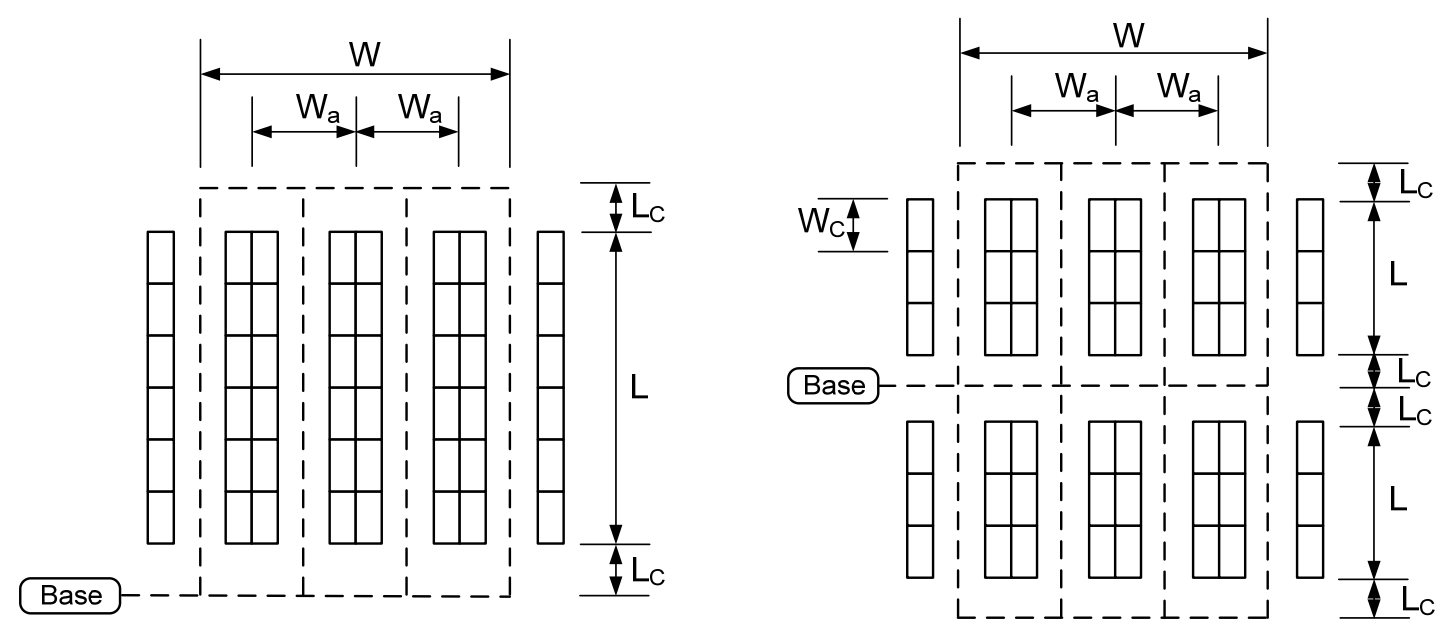

Figure 6: Parameters in a single block layout(left) and in a double block layout(right)

It is assumed that the basic distance $s_{B}$ which generally depends only on the storage system layout, is known. The within-aisles distance $s_{W}$ and the across-aisles distance $s_{A}$, on the other hand, depend on the pick path routing strategy of the picker.

As routing strategy, the Return Strategy With and Without Repetition, the Traversal Strategy With and Without Skips, the Midpoint Heuristic as well as the Largest-Gap Heuristic can be differentiated. In the following sections, the method for estimating the travel distance using the Return Strategy With and Without Repetition, the Midpoint Heuristic and the Traversal Strategy With and Without Skips is described. 


\subsection{Estimation of the within-aisle distance}

The within-aisles distance describes the distance to be covered by an order picker within the aisles while picking $n$ items. The $n$ items are distributed across $N_{A}$ aisles. The within-aisle distance depends on the routing strategy used. Additionally the constant length in front of the aisle $\mathrm{L}_{\mathrm{C}}$ has to be considered [StH08].

\subsubsection{Return Strategy With Repetitions}

If the Return Strategy With Repetition is used, the average distance that the picker has to travel into an aisle for one item is equal to the sum of the constant length $L_{C}$ and the expected value of the standardized probability density function $f(x)$ multiplied with the length of the aisle $L$.

Since the probability density function is the same for all aisles, the expected values are also the same. Considering the way inside the aisle and back, the travel distance $s_{W}$ for picking $n$ items is described by

$$
s_{W}=2 \cdot n \cdot\left[L_{C}+L \cdot \int_{0}^{\infty} x \cdot f(x) d x\right]
$$

If the access frequency is equal to a uniform distribution with $a=0$ and $b=1$, then the travel distance $s_{W}$ by using (1) in (7) turns into

$$
s_{W}=2 \cdot n \cdot\left[L_{C}+\frac{1}{2} \cdot L\right] \quad \text { (uniform distribution) }
$$

If the access frequency of the bin locations is modeled by an exponential distribution, the within-aisle distance using (3) in (7) resolves to

$$
S_{W}=2 \cdot n \cdot\left[L_{C}+L \cdot \frac{1}{\lambda}\right] \quad \text { (exponential distribution) }
$$

These equations are valid in the single block layout as well as in the double block layout.

\subsubsection{Return Strategy Without Repetition}

Depending on the number of orderlines per aisle $r$, there are different average distances $s_{r}$ which have to be covered by the picker in each aisle. When the average distance $s_{r}$ is multiplied by the probability of its occurrence, the within-aisle distance $s_{W}$ is generally calculated as follows:

$$
s_{W}=\sum_{r=1}^{n} p_{a l l}(r) \cdot s_{r}
$$

If $p_{i}(r)$ is the probability to find $r$ orderlines in aisle $i$, the relation to the overall probability $p_{\text {all }}(r)$ is

$$
p_{\text {all }}(r)=\sum_{i=1}^{N_{\mathrm{A}}} p_{i}(r)
$$

If the access frequencies are the same for each aisle the probability is

$$
p_{1}(r)=p_{2}(r)=\ldots=p_{N_{A}}(r)=p(r)
$$

The probability of the distance $s_{r}$ thus can be described by

$$
p_{\text {all }}(r)=N_{A} \cdot p(r)
$$

The within-aisles distance $s_{W}$ can be calculated by using (13) in (10) 


$$
s_{W}=N_{A} \cdot \sum_{r=1}^{n} p(r) \cdot s_{r}
$$

To accomplish this, the distance $s_{r}$ which has to be covered in an aisle during the picking of $r$ orderlines must be calculated. In the second step, the respective probabilities are determined. According to the pick path routing strategy applied, there are different approaches to determine $s_{r}$.

In the Return Strategy, the average distance to be covered in an aisle during the picking of $r$ orderlines depends only on the bin location with the maximum distance to the aisle front.

When the density function $f(x)$ of the access frequencies versus the distance from the aisle front is known, the random variable $X$ can be defined as the maximum distance to be covered during the picking of $r$ orderlines. The distribution function of the maximum values $X_{(r)}(x)$ can be described by the following equation according to the order statistic rules [Gen04, Mod73]

$$
F_{X(r)}(x)=[F(x)]^{r}
$$

The density function $f_{X(r)}(x)$ of $X_{(r)}(x)$ corresponds to the derivation of the distribution function

$$
f_{X(r)}=r \cdot[F(x)]^{r-1} \cdot f(x)
$$

The expected value of $f_{X(r)}(x)$ represents the expected distance that an order picker has to go into an aisle to pick $r$ orderlines without repetitions according to the Return Strategy. Since the order picker's path back is the same and the constant length $L_{C}$ is considered, the following equation applies

$$
s_{r}=2 \cdot\left(L_{C}+E\left(X_{(r)}\right)\right)
$$

The general definition of the expected value and (16) results in

$$
\begin{aligned}
E\left(X_{(r)}\right) & =\int_{0}^{\infty} x \cdot f_{X(r)}(x) d x \\
& =\int_{0}^{\infty} x \cdot r \cdot f(x) \cdot[F(x)]^{r-1} d x
\end{aligned}
$$

Alternatively, the expected value can be determined as follows

$$
\begin{aligned}
E\left(X_{(r)}\right) & =\int_{0}^{\infty}\left[1-F_{X(r)}(x)-F_{X(r)}(-x)\right] d x \\
& =\int_{0}^{\infty}[1-[F(x)]^{r}-\underbrace{[F(-x)]^{r}}_{=0}] d x \\
& =\int_{0}^{\infty} 1-[F(x)]^{r} d x
\end{aligned}
$$

The resulting equation for the average distance $s_{r}$ covered during the non-repetitive picking of $r$ orderlines according to the Return Strategy using (18) in (17) turns into

$$
s_{r}=2 \cdot\left(L_{C}+\int_{0}^{\infty} x \cdot r \cdot f(x) \cdot[F(x)]^{r-1} d x\right)
$$

Or by using (19) in (17) turns into 


$$
s_{r}=2 \cdot\left(L_{C}+\int_{0}^{\infty} 1-[F(x)]^{r} d x\right)
$$

When the known distances have to be covered within an aisle to pick the orderlines, the Laplace probability $p(r)$ must be determined for the case that exactly $r$ items out of a total of $n$ items are picked in an aisle. This can be described by an urn model without return. The urn holds $M_{A}$ black balls and $M-M_{A}$ white balls. The black balls represent the stored items in the respective aisle and the white balls the items in all other aisles. A sample $n$ is taken from the urn without return. To determine the probability that the sample $n$ contains exactly $r$ black balls, it is required to count all possible combinations of $r$ black balls. Since no ball is returned and the sequence is not considered, the number of possible combinations is the binomial coefficient of $M_{A}$ and $r\left(\begin{array}{l}M_{A} \\ r\end{array}\right)$.

The number of possible combinations of $n-r$ white balls is determined in the same way, i.e. $\left(\begin{array}{l}M-M_{A} \\ n-r\end{array}\right)$. Since all black ball combinations can be combined with any white ball combination the probability to pick $r$ black balls in $n$ picks is $\left(\begin{array}{l}M_{A} \\ r\end{array}\right) \cdot\left(\begin{array}{l}M-M_{A} \\ n-r\end{array}\right)$. To determine the Laplace probability the number of 'favourable' events is divided by the number of all combinations, i.e. by $\left(\begin{array}{l}M \\ n\end{array}\right)$ and the result equals the probability $p(r)$

$$
p(r)=\frac{\left(\begin{array}{l}
M_{A} \\
r
\end{array}\right) \cdot\left(\begin{array}{l}
M-M_{A} \\
n-r
\end{array}\right)}{\left(\begin{array}{l}
M \\
n
\end{array}\right)} \quad r=0,1,2, \ldots
$$

The probability to pick $r$ orderlines in an aisle corresponds to the hyper geometrical distribution. By using (20) and (22) in (14) the distance in an aisle $s_{W}$ is generally calculated as follows

$s_{W}=2 \cdot N_{A} \cdot \sum_{r=1}^{n}\left[\frac{\left(\begin{array}{l}M_{A} \\ r\end{array}\right) \cdot\left(\begin{array}{l}M-M_{A} \\ n-r\end{array}\right)}{\left(\begin{array}{l}M \\ n\end{array}\right)} \cdot\left(L_{C}+\int_{0}^{\infty} x \cdot r \cdot f(x) \cdot[F(x)]^{r-1} d x\right)\right]$

In case of the access frequency being uniformly distributed over the complete length $L$ of the aisle, in (1) the parameters $a$ and $b$ apply to $a=0$ and $b=1$. So (20) simplifies to

$$
S_{r}=2 \cdot\left(L_{C}+L \cdot \int_{0}^{1} x \cdot r \cdot \frac{1}{1} \cdot\left[\frac{x}{1}\right]^{r-1} d x\right)
$$




$$
\begin{aligned}
& =2 \cdot\left(L_{C}+L \cdot \frac{r}{1^{r-1}} \cdot \int_{0}^{1} x \cdot x^{r-1} d x\right) \\
& =2 \cdot\left(L_{C}+L \cdot \frac{r}{1^{r}} \int_{0}^{1} x^{r} d x\right) \\
& =2 \cdot\left(L_{C}+L \cdot \frac{1}{1^{r}} \cdot \frac{r}{r+1} \cdot\left[x^{r+1}\right]_{0}^{1}\right) \\
& =2 \cdot\left(L_{C}+L \cdot \frac{r}{r+1} \cdot \frac{1^{r+1}}{1^{r}}\right) \\
& =2 \cdot\left(L_{C}+L \cdot \frac{r}{r+1}\right)
\end{aligned}
$$

By using (22) and (25) in (10) the within-aisle distance $\mathrm{s}_{W}$ is

$$
s_{W}=2 \cdot N_{A} \cdot \sum_{r=1}^{n}\left[\frac{\left(\begin{array}{l}
M_{A} \\
r
\end{array}\right) \cdot\left(\begin{array}{l}
M-M_{A} \\
n-r
\end{array}\right)}{\left(\begin{array}{l}
M \\
n
\end{array}\right)} \cdot\left(L_{C}+L \cdot \frac{r}{r+1}\right)\right]
$$

When the access frequency of the bin locations is modeled by a standard exponential distribution, as described above, the average within-aisles distances using (4) in (21) are

$$
\begin{aligned}
S_{r} & =2 \cdot\left(L_{C}+L \cdot \int_{0}^{\infty} 1-[F(x)]^{r} d x\right) \\
& =2 \cdot\left(L_{C}+L \cdot \int_{0}^{\infty} 1-\left(1-e^{-\lambda x}\right)^{r} d x\right) \\
& =2 \cdot\left(L_{C}+L \cdot \lim _{z \rightarrow \infty} \int_{0}^{z} 1-\left(1-e^{-\lambda x}\right)^{r} d x\right) \\
& =2 \cdot\left(L_{C}+L \cdot \lim _{z \rightarrow \infty}\left[\frac{1}{\lambda} \sum_{k=1}^{r} \frac{1}{k}\left(1-e^{-\lambda z}\right)^{k}\right]\right) \\
& =2 \cdot\left(L_{C}+\frac{L}{\lambda} \sum_{k=1}^{r} \frac{1}{k}\right)
\end{aligned}
$$

Using (22) and (27) in (10) the within-aisles distance is

$$
s_{W}=2 \cdot N_{A} \cdot \sum_{r=1}^{n}\left[\frac{\left(\begin{array}{l}
M_{A} \\
r
\end{array}\right) \cdot\left(\begin{array}{l}
M-M_{A} \\
n-r
\end{array}\right)}{\left(\begin{array}{l}
M \\
n
\end{array}\right)} \cdot\left(L_{C}+\frac{L}{\lambda} \sum_{k=1}^{r} \frac{1}{k}\right)\right]
$$

These equations are valid in the single block layout as well as in the double block layout. 


\subsubsection{Midpoint Heuristic}

If the picker moves through the storage according to the midpoint heuristic, the within-aisle distance can be calculated basically in the same way as the Return Strategy Without Repetition.

Using this strategy, the picker has to change the aisles minimum once on the rear side of the storage. Thus, the order picker covers at least a constant section of the distance

$s_{w}^{C}=2 \cdot\left(2 \cdot L_{C}+L\right)$

independent from the number of orderlines to be picked. Only if all items are located in one aisle, this section is shorter. However, this is an exception, which is neglected in the calculations. Therefore the whole within-aisle travel distance which is covered during one picking tour, consists of a constant section and a variable section.

Because two aisles are already considered for the constant distance calculation, the number of aisles to be considered for the variable section must be reduced by two units.

The order picker enters the remaining aisles $\left(N_{A^{-}}-2\right)$ once on the front side and once on the rear side and only moves up to the middle of the aisle. For this reason, all remaining aisles are regarded as two independent aisles with half length and half number of items. If $N_{A}{ }^{*}$ is defined as number of aisles to be considered in the variable section and $L^{*}$ und $M_{A}{ }^{*}$ as the according length and number of items per aisle, the whole within-aisle travel distance is obtained by the addition of (29) and (23)

$$
\begin{aligned}
s_{W}= & 2 \cdot\left(2 \cdot L_{C}+L\right)+2 \cdot N_{A}^{*} \cdot \\
& \sum_{r=1}^{n}\left[\frac{\left(\begin{array}{l}
M_{A}^{*} \\
r
\end{array}\right) \cdot\left(\begin{array}{l}
M-M_{A}^{*} \\
n-r
\end{array}\right)}{\left(\begin{array}{l}
M \\
n
\end{array}\right)} \cdot\left(L_{C}+L^{*} \cdot \int_{0}^{\infty} x \cdot r \cdot f(x) \cdot[F(x)]^{r-1} d x\right)\right]
\end{aligned}
$$

When replacing $N_{A}{ }^{*}, M_{A}{ }^{*}$ and $L^{*}$ in (30) by

$$
\begin{aligned}
& N_{A}^{*}=2 \cdot\left(N_{A}-2\right) \\
& M_{A}^{*}=\frac{M_{A}}{2} \\
& L^{*}=\frac{L}{2}
\end{aligned}
$$

the general relation for the calculation of the within-aisle travel distance when applying the Midpoint Heuristic in a one block aisle layout can be obtained by

$$
\begin{aligned}
S_{W}=2 \cdot\left(2 \cdot L_{C}+L\right)+4 \cdot\left(N_{A}-2\right) \cdot \\
\sum_{r=1}^{n}\left[\frac{\left(\begin{array}{l}
\frac{M_{A}}{2} \\
r
\end{array}\right) \cdot\left(\begin{array}{l}
M-\frac{M_{A}}{2} \\
n-r
\end{array}\right)}{\left(\begin{array}{l}
M \\
n
\end{array}\right)} \cdot\left(L_{C}+\frac{L}{2} \cdot \int_{0}^{\infty} x \cdot r \cdot f(x) \cdot[F(x)]^{r-1} d x\right)\right]
\end{aligned}
$$


In the case of a double block layout the constant section $s_{W}{ }^{C}$ doubles to

$s_{w}^{C}=4 \cdot\left(2 \cdot L_{C}+L\right)$

because the picker additionally has to cross the central aisle for reaching the rear side of the second block. Consequently, $N_{A}{ }^{*}$ is

$N_{A}^{*}=2 \cdot\left(N_{A}-4\right)$

The within-aisle travel distance in the double block layout therefore is determined by the relation

$$
\begin{aligned}
& S_{W}=4 \cdot\left(2 \cdot L_{C}+L\right)+4 \cdot\left(N_{A}-4\right) \cdot \\
& \sum_{r=1}^{n}\left[\frac{\left(\begin{array}{l}
\frac{M_{A}}{2} \\
r
\end{array}\right) \cdot\left(\begin{array}{l}
M-\frac{M_{A}}{2} \\
n-r
\end{array}\right)}{\left(\begin{array}{l}
M \\
n
\end{array}\right)} \cdot\left(L_{C}+\frac{L}{2} \cdot \int_{0}^{\infty} x \cdot r \cdot f(x) \cdot[F(x)]^{r-1} d x\right)\right]
\end{aligned}
$$

If the access frequency of one item is considered during the bin assignment and the Midpoint Heuristic is applied, it is reasonable to place the items with the highest access frequencies at the beginning and at the end of the aisles. The access frequency can be approached by the exponential distribution up to the middle of the aisle.

Figure 7 shows the exponential distribution in case of the return Strategy as well as the related exponential distribution for the Midpoint Heuristic.
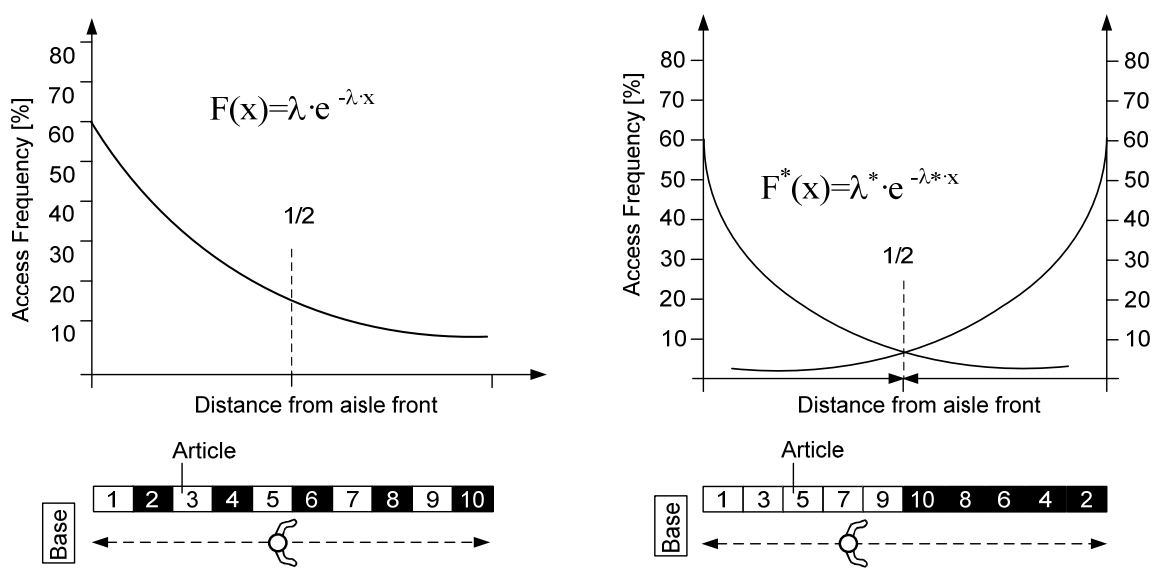

Figure 7: Access frequency in an aisle for different routing strategies; Return Strategy (left) and Midpoint Heuristic (right)

For the calculation of the within-aisle distance, the expected value $\mathrm{E}(\mathrm{X})^{*}$ has to be known. This value can be derived from the bin assignment of the items.

Thus, using the Midpoint Heuristic the within-aisle distance depends on the layout and the distribution density function.

The following cases show the different equations for the diverse combinations of layouts and density functions. 
Case 1: Single block layout / uniform distribution:

$s_{W}=2 \cdot\left(2 \cdot L_{C}+L\right)+4 \cdot\left(N_{A}-2\right) \cdot$

$$
\sum_{r=1}^{n}\left[\frac{\left(\begin{array}{l}
\frac{M_{A}}{2} \\
r
\end{array}\right) \cdot\left(\begin{array}{l}
M-\frac{M_{A}}{2} \\
n-r
\end{array}\right)}{\left(\begin{array}{l}
M \\
n
\end{array}\right)} \cdot\left(L_{C}+\frac{L}{2} \cdot \frac{r}{r+1}\right)\right]
$$

Case 2: Single block layout / exponential distribution:

$s_{W}=2 \cdot\left(2 \cdot L_{C}+L\right)+4 \cdot\left(N_{A}-2\right) \cdot$

$$
\sum_{r=1}^{n}\left[\frac{\left(\begin{array}{l}
\frac{M_{A}}{2} \\
r
\end{array}\right) \cdot\left(\begin{array}{l}
M-\frac{M_{A}}{2} \\
n-r
\end{array}\right)}{\left(\begin{array}{l}
M \\
n
\end{array}\right)} \cdot\left(L_{C}+\frac{L}{2} \cdot \frac{1}{\lambda^{*}} \cdot \sum_{k=1}^{r} \frac{1}{k}\right)\right]
$$

For the double block layout analogously applies.

Case 3: Double block layout / uniform distribution

$s_{W}=4 \cdot\left(2 \cdot L_{C}+L\right)+4 \cdot\left(N_{A}-4\right) \cdot$

$$
\sum_{r=1}^{n}\left[\frac{\left(\begin{array}{l}
\frac{M_{A}}{2} \\
r
\end{array}\right) \cdot\left(\begin{array}{l}
M-\frac{M_{A}}{2} \\
n-r
\end{array}\right)}{\left(\begin{array}{l}
M \\
n
\end{array}\right)} \cdot\left(L_{C}+\frac{L}{2} \cdot \frac{r}{r+1}\right)\right]
$$

Case 4: Double block layout / exponential distribution:

$$
s_{W}=4 \cdot\left(2 \cdot L_{C}+L\right)+4 \cdot\left(N_{A}-4\right) \cdot
$$

$$
\sum_{r=1}^{n}\left[\frac{\left(\begin{array}{c}
\frac{M_{A}}{2} \\
r
\end{array}\right) \cdot\left(\begin{array}{c}
M-\frac{M_{A}}{2} \\
n-r
\end{array}\right)}{\left(\begin{array}{l}
M \\
n
\end{array}\right)} \cdot\left(L_{C}+\frac{L}{2} \cdot \frac{1}{\lambda^{*}} \cdot \sum_{k=1}^{r} \frac{1}{k}\right)\right]
$$




\subsubsection{Traversal Strategy With Skips}

When the order picker works according to the Traversal Strategy and skips those aisles where no items have to be picked, the aisle distance can be calculated with (10). The distances then always correspond to the total aisle length $L_{C}+L$, i.e.

$$
s_{r}(1)=s_{r}(2)=s_{r}(3)=s_{r}(4)=\ldots=s_{r}=2 \cdot L_{C}+L
$$

Thus, (10) is simplified as follows

$$
s_{W}=N_{A} \cdot\left[2 \cdot L_{C}+L\right] \cdot \sum_{r=1}^{n} p(r)
$$

With the constraint

$$
\begin{gathered}
\sum_{r=0}^{n} p(r)=1 \\
\Leftrightarrow \sum_{r=1}^{n} p(r)+p(0)=1 \\
\Leftrightarrow \sum_{r=1}^{n} p(r)=1-p(0)
\end{gathered}
$$

and by using (45) in (43) the result is

$$
s_{W}=N_{A} \cdot\left[2 \cdot L_{C}+L\right] \cdot(1-p(0))
$$

With (12) $p(0)$ is

$$
p(0)=\frac{\left(\begin{array}{c}
M_{A} \\
0
\end{array}\right) \cdot\left(\begin{array}{c}
M-M_{A} \\
n-0
\end{array}\right)}{\left(\begin{array}{l}
M \\
n
\end{array}\right)}=\frac{\left(\begin{array}{c}
M-M_{A} \\
n
\end{array}\right)}{\left(\begin{array}{l}
M \\
n
\end{array}\right)}
$$

By using (47) in (46) the average within-aisles distance to be covered in a Traversal Strategy With Skips is

$$
S_{W}=N_{A} \cdot\left[2 \cdot L_{C}+L\right] \cdot\left[1-\frac{\left(\begin{array}{c}
M-M_{A} \\
n
\end{array}\right)}{\left(\begin{array}{l}
M \\
n
\end{array}\right)}\right]
$$

\subsubsection{Traversal Strategy Without Skips}

Using the Traversal Strategy Without Skips, the picker has to pass each aisle at least once. When the number of aisles is odd the last aisle is passed twice. The within-aisle distance therefore depends on the number of aisles, i.e.

$$
s_{W}= \begin{cases}N_{A} \cdot\left(2 \cdot L_{C}+L\right) & \text { for } N_{A} \text { even } \\ \left(N_{A}+1\right) \cdot\left(2 \cdot L_{C}+L\right) & \text { for } N_{A} \text { odd }\end{cases}
$$




\subsection{Estimation of the across-aisles distance}

Two aspects have to be considered when calculating the across-aisles distance. If the order picker follows the Traversal Strategy Without Skips, the aisle changing distance is twice the distance $W$ between the first and the last aisle if the base is located outside the order picking system (see Figure 6), i.e.

$$
s_{A}=2 \cdot W \quad \text { (without skips) }
$$

If the order picker knows at the beginning of his tour where the items are located, he can skip all unused aisles. The across-aisles distance then corresponds to the distance between the most remote aisle and the path back to the base. Here, the approach in (16) is used whereas $r$ has to be replaced by $n$. The equation is

$$
s_{A}=2 \cdot \int_{0}^{\infty} x \cdot n \cdot f(x) \cdot[F(x)]^{n-1} d x
$$

By using (1) and (2) in (51) the across-aisle distance for the uniform distribution results in

$$
s_{A}=2 \cdot \int_{a}^{b} x \cdot n \cdot \frac{1}{b-a} \cdot\left[\frac{x-a}{b-a}\right]^{n-1} d x
$$

When the access frequency across all aisles is assumed to be equal, the following equation applies for the parameters $a=0$ and $b=W$.

$$
\begin{array}{ll}
s_{A}=2 \cdot \int_{0}^{W} x \cdot n \cdot \frac{1}{W} \cdot\left[\frac{x}{W}\right]^{n-1} d x & \quad \text { (with skips) } \\
s_{A}=2 \cdot \frac{n}{n+1} \cdot W
\end{array}
$$

The methodology for estimating the across-aisle distance in a single block layout is equal to the double block layout. If the base located inside the order picking system and $n>1$ the across-aisle distance is also equal in both systems [Sch96].

\section{Validation of the travel distance calculation method using simulation}

The presented estimation method for the determination of travel distances in a low-level picker-to-part orderpicking system was developed on the basis of several assumptions and restrictions. As a result of these simplifications, the calculated values may not exactly match the values of the real system. For using the estimation method during the planning stage, the accuracy level must be defined.

A simulation model for the representation of different warehouse dimensions has been developed for determining the accuracy. With the simulation model the stochastic effects of real systems can be considered and the exact travel distances covered by an order picker when processing a work assignment can be determined experimentally. By comparing the estimated values with results of the simulation, the accuracy of the calculations can be determined. A precise description of the validation of the simulation model is given in [Sad07].

Besides this, the results of the common calculation methods are also compared with the results of the simulation model.

\subsection{Basis}

The three systems examined vary in the size of their assortments, their bin location width and the number of aisles. Regarding the number of aisles and the assortment size, the parameters were chosen to cover the most common range of real world systems.

In the simulation model and during the calculations, the SKU access frequency is realized by means of an exponential distribution with a specified expected value $E(X)$. This ensures that identical preconditions are obtained for the analytical determination of the aisle distances and the travel distances across-aisles, as well as for those values determined during the simulation. Table 1 provides an overview of the data of the three systems. 
Table 1: Inspected order picking systems

\begin{tabular}{c|c|c|c|c|c|c|c} 
System & $\mathrm{M}$ & $\mathrm{N}_{\mathrm{A}}$ & $\mathrm{W}_{\mathrm{C}}[\mathrm{m}]$ & $\mathrm{L}[\mathrm{m}]$ & $\mathrm{L}_{\mathrm{C}}[\mathrm{m}]$ & $\mathrm{W}_{\mathrm{A}}[\mathrm{m}]$ & $\mathrm{B}[\mathrm{m}]$ \\
\hline 1 & 300 & 5 & 1,2 & 36 & 1 & 3,5 & 14 \\
\hline 2 & 960 & 8 & 0,6 & 36 & 1 & 3,5 & 24,5 \\
\hline 3 & 3600 & 10 & 0,2 & 36 & 1 & 3,5 & 31,5
\end{tabular}

The within-aisle distance and the across-aisles distance are determined analytically and by simulation during the following investigation for a fixed specified number of orderlines per work assignment. The investigation is comprised of orders with $n \in\{1 \ldots .50\}$ orderlines per order. The within-aisle distances are investigated for the Traversal Strategy With Skips (TPwS), for the Return Strategy With Repetition (RPwR), and for the Return Strategy Without Repetition (RPoR).

The simulated and analytically determined travel distances are graphically represented, depending on the number of orderlines per order. Moreover, the simulation covers the percentile deviation of the different calculation methods.

Since the results of the three investigated systems draw the same conclusions, only the results of system 2 are presented and explained in detail. For the results of systems 1 and 3, please refer to the appendix.

\subsection{Representation of the results}

If the SKU access frequency is considered during the bin assignment process, the access frequency over the distance from the aisle front corresponds with the density function describing the access frequency of all items. During the validation, an expected value $\mathrm{E}(\mathrm{X})$ of 0.1244 was assumed for the standardized exponential distribution, based on the fact that in this case $20 \%$ of the items required account for $80 \%$ of the bin accesses. When applying the Traversal Strategy With Skips, the absolute values shown in Figure 8 are obtained for the within-aisle distances of system 2. The percentile deviations in comparison to the results of the simulation are represented in Figure 9.

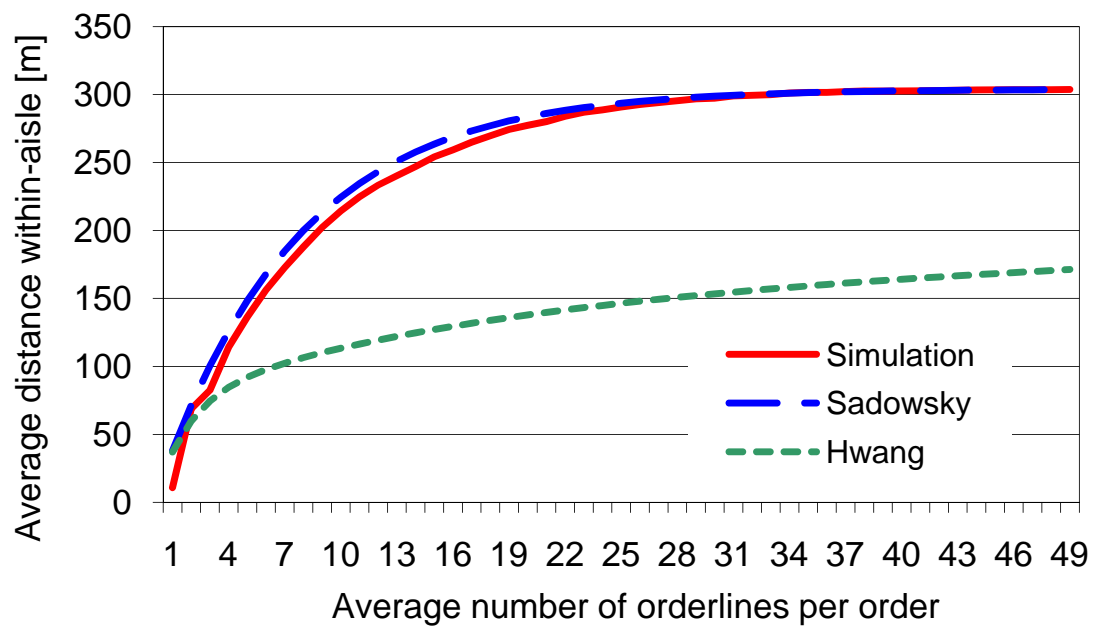

Figure 8: Within-aisle distance; System 2; exponentially distributed access rate - Traversal Strategy With Skips 


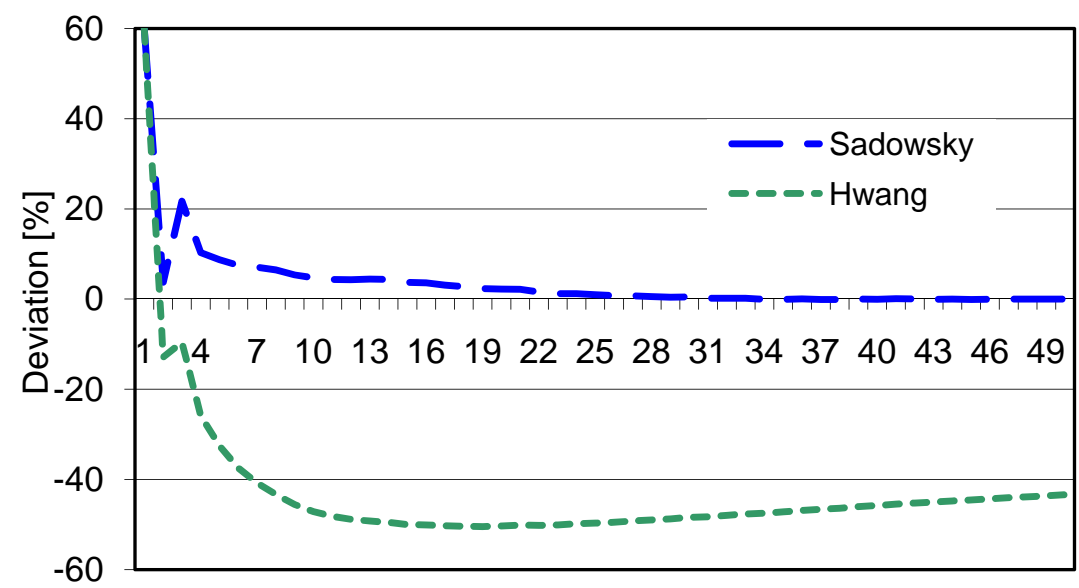

Average number of orderlines per order

Figure 9: Percentile deviation of the calculated within-aisle distance from the simulation; System 2; exponentially distributed access rate - Traversal Strategy With Skips

Both calculation methods produce major deviations from the simulation data for $n=1$. This is due to the fact that the picker does not cover the travel distance of a Traversal Strategy when picking a single item order. In reality, the picker enters an aisle, picks the required item, simultaneously finishing the order, and then travels back to the aisle front. That means that the actual travel distance of the order picker is equivalent to the pick path of the Return Strategy. A comparison of the aisle distances for the Traversal Strategy and the Return Strategy for $n=1$, which are determined during the simulation, confirms this statement. If the order consists of two orderlines, the probability that both orderlines are located in different aisles is very high. For this reason, two aisles are passed through in most cases. In this case, the results of the new estimation method vary by only $3.73 \%$ from the simulation results.

In case of three orderlines per order, there is a similar effect as in the single item order example. In this case the deviation has a lower impact since the first two orderlines have been picked according to the Traversal Strategy, and only the pick path of the third item complies with the Return Strategy.

Nevertheless, the deviation still amounts to $21.73 \%$. The higher the quantity of orderliness per order, the lower the deviation rate since the total distance has become longer.

With five orderlines per order, the rate of deviation falls to $10 \%$. Beginning with 10 orderlines per order the $5 \%$ mark will not be reached. If there are more than 25 orderlines per order, the deviation will fall to less than $1 \%$.

Hwang's estimations show, at first, a similar shape. However, if there are more than 3 orderlines per order, the deviation rate is decreasing into the negative range. At nine orderlines the deviation rate per order reaches $-45 \%$. This deviation remains constant at this level even if there are more orderlines.

Regarding to the Return Strategy, both estimation methods largely match the simulation data (Figure 10). Compared with the simulation (Figure 11) both methods have a max. deviation of $1.5 \%$ (Sadowsky), respectively $8 \%$ (Hwang). 


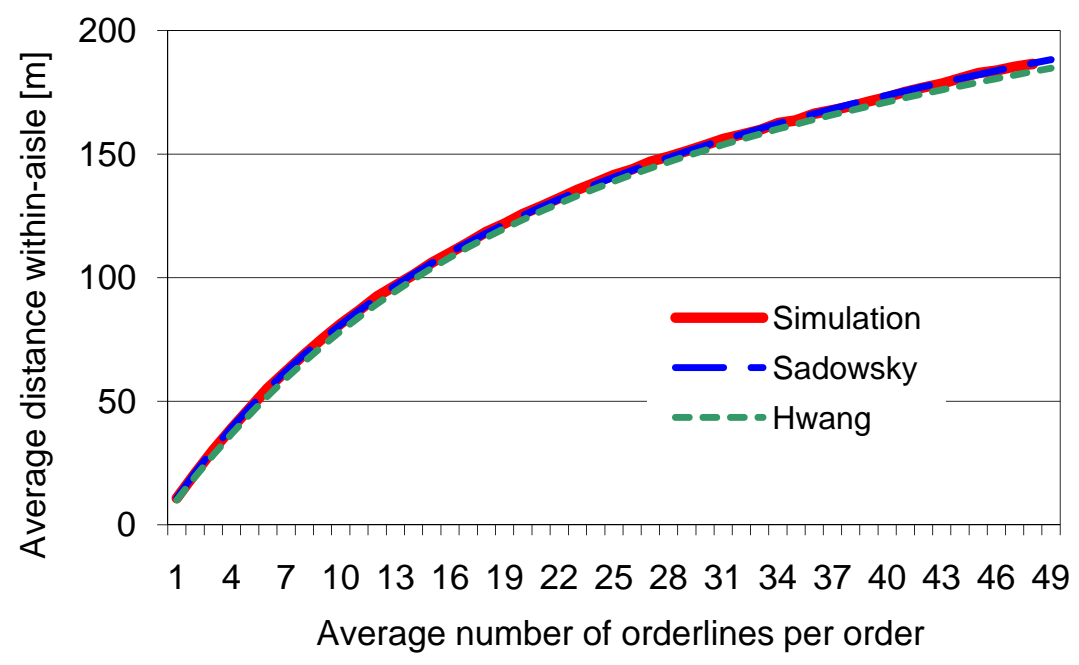

Figure 10: Within-aisle distance; System 2; exponentially distributed access rate - Return Strategy Without Repetition

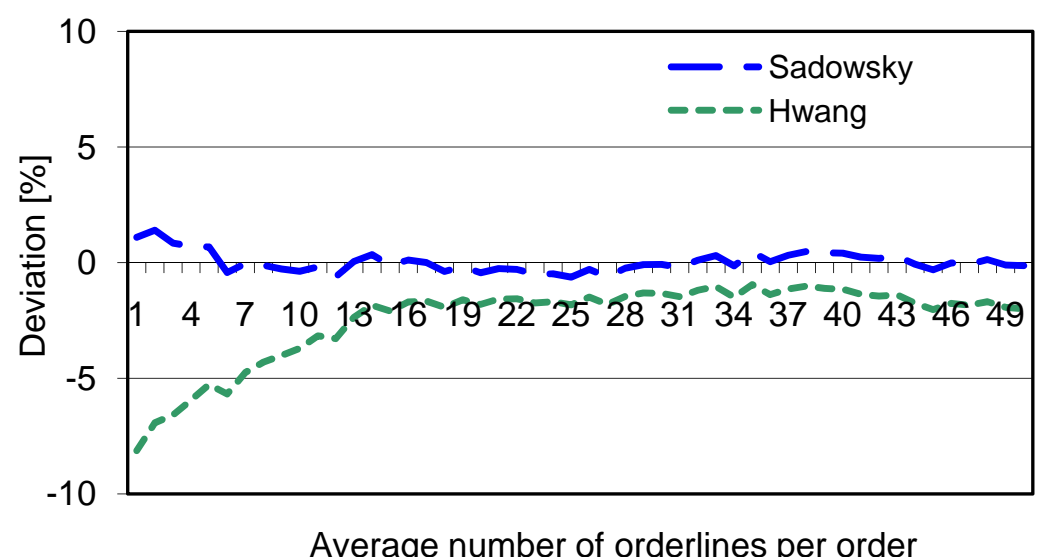

Figure 11:Deviation oft he calculated within-aisle distance from the simulation; System 2; exponentially distributed access rate - Return Strategy Without Repetition

If more than five orderlines are handled per order the deviation drops below $0.5 \%$ using the new estimation method, while Hwang's method drops below the 5\%. Hwang does not mention any computation equation for the Return Strategy With Repetition. For two orderlines the results deviate by a maximum of $1.3 \%$. With four orderlines per order the deviation falls below 1\% (Figure 13). 


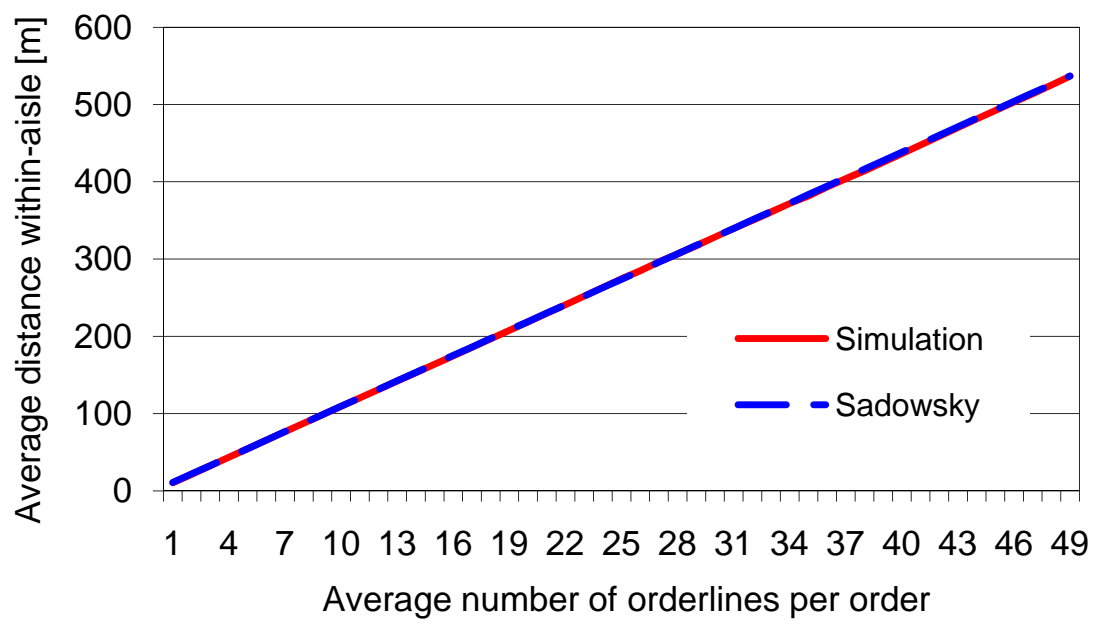

Figure 12: Within-aisle distance; System 2; exponentially distributed access rate - Return Strategy With Repetition

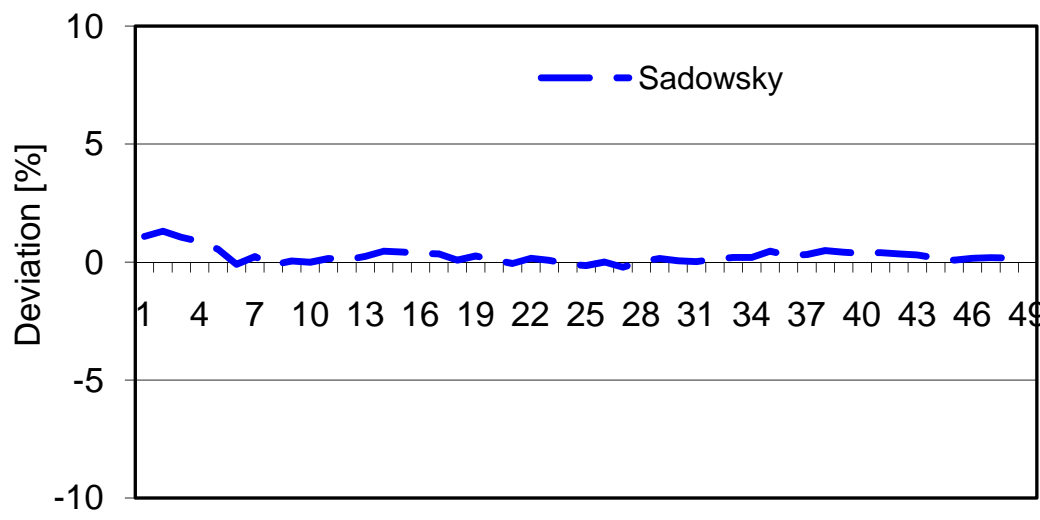

Average number of orderlines per order

Figure 13:Deviation of the calculated within-aisle distance from the simulation; System 2; exponentially distributed access rate - Return Strategy With Repetition

Figure 14 shows the calculated within-aisle distances and the values for the Midpoint Heuristic determined by simulation. The deviations are shown in Figure 15.

In case of a low number of orderliness, the within-aisle distance determined for the Midpoint Heuristic according to the new calculation method considerably deviates at the beginning. This is due to the constant part of the distance. If there are more than four orderlines, the deviation drops below $10 \%$. If more than ten orderlines are picked per order, the deviation is less than $5 \%$.

The curve progression for Hwang's calculation method is very similar for low numbers of orderlines. If there are two orderlines per order, the deviation falls to $-2.47 \%$. With an increasing number of orderlines per order, the deviation rises up to $11 \%$. 


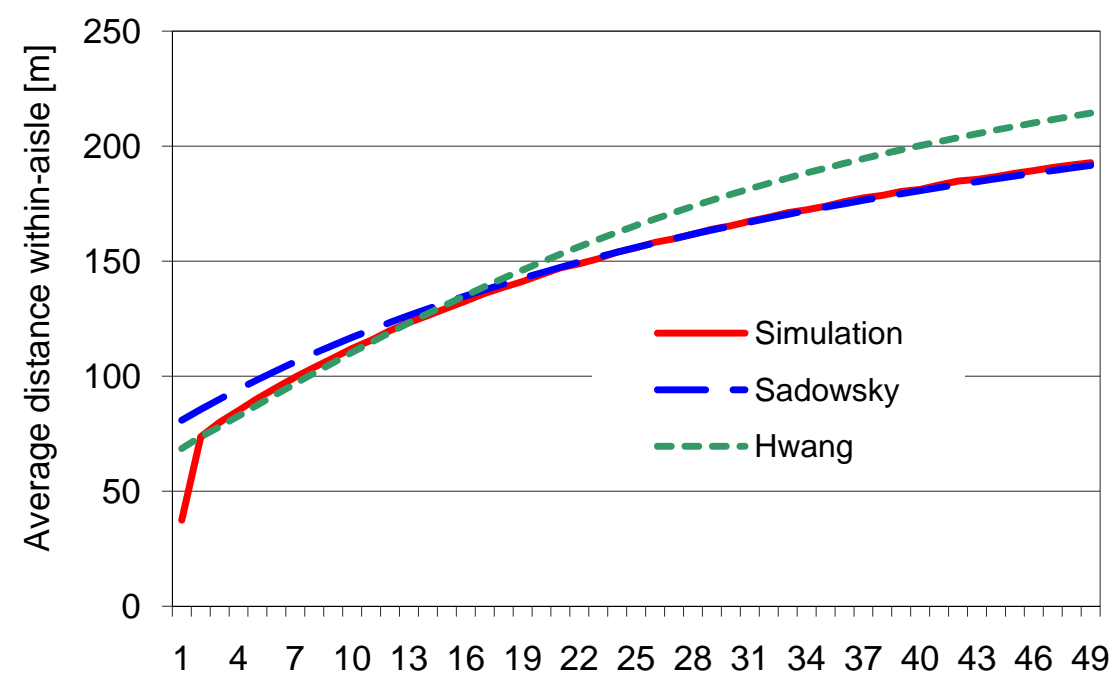

Average number of orderlines per order

Figure 14: Within-aisle distance; System 2; exponentially distributed access rate - Midpoint Heuristic

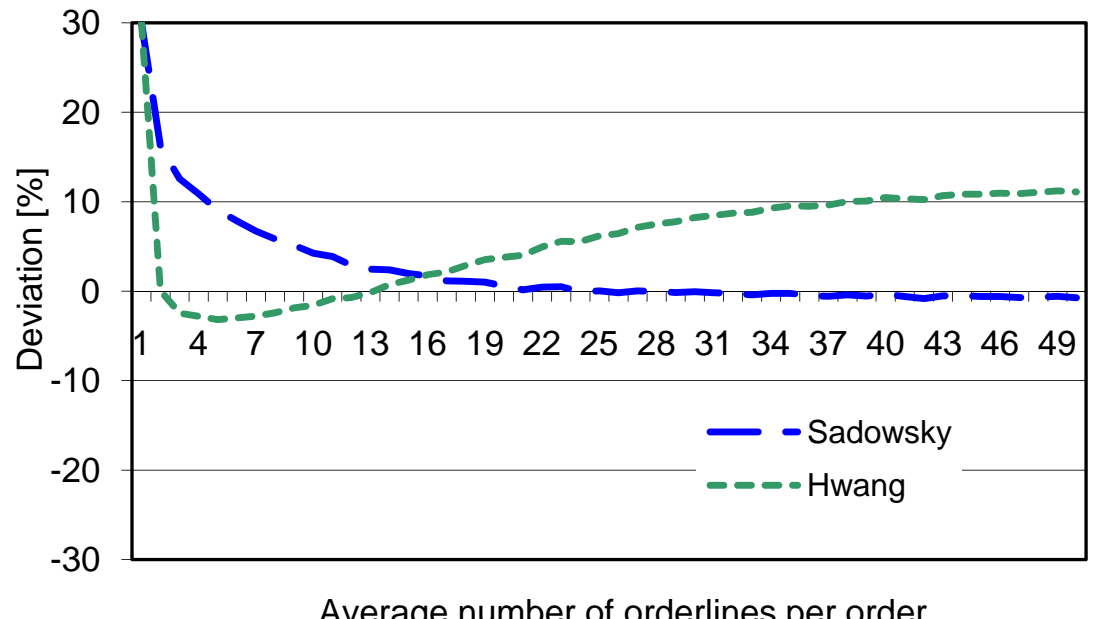

Figure 15: Deviation of the calculated within-aisle distance from the simulation; System 2; exponentially distributed access rate - Midpoint Heuristic

The length of the across-aisle distance does not depend on the pick path routing strategy. The simulation process thereby provides identical results for each pick path routing strategy regarding the across-aisle distance. The consideration of the density function of the access frequency within the aisles is only relevant to the calculations of Hwang. All other methods do not take this distribution into account.

Figures 16 and 17 show the average across-aisle distances and the percentile deviation from the simulation in the case of a uniformly distributed access rate within the aisles. 


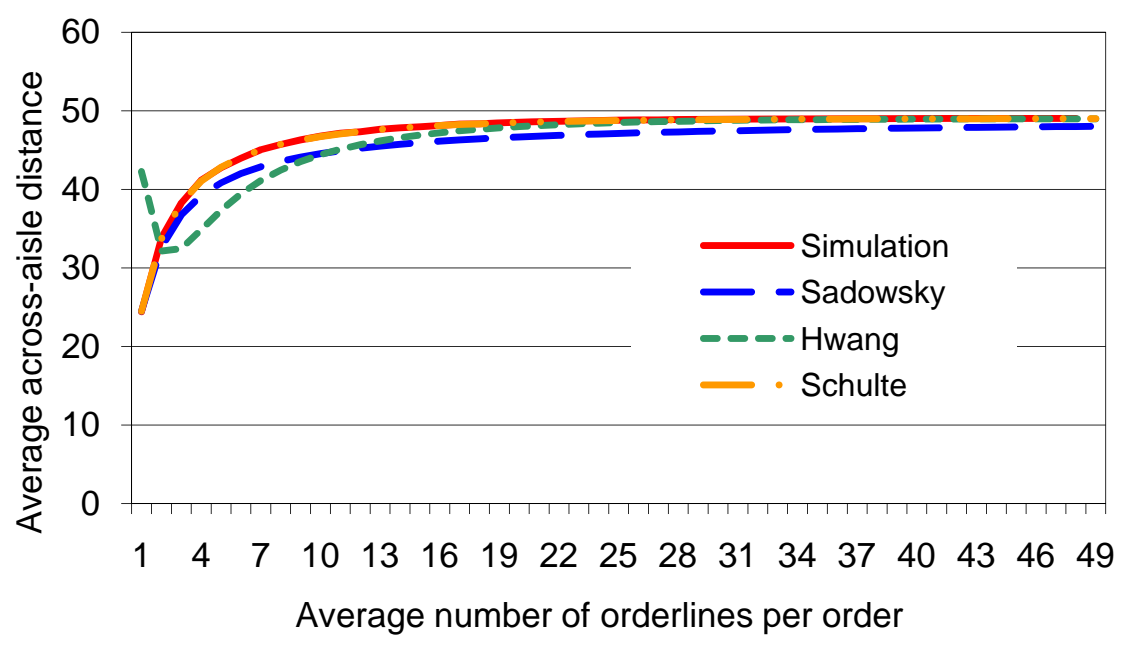

Figure 16: Across-aisle distance; System 2; uniformly distributed access frequency

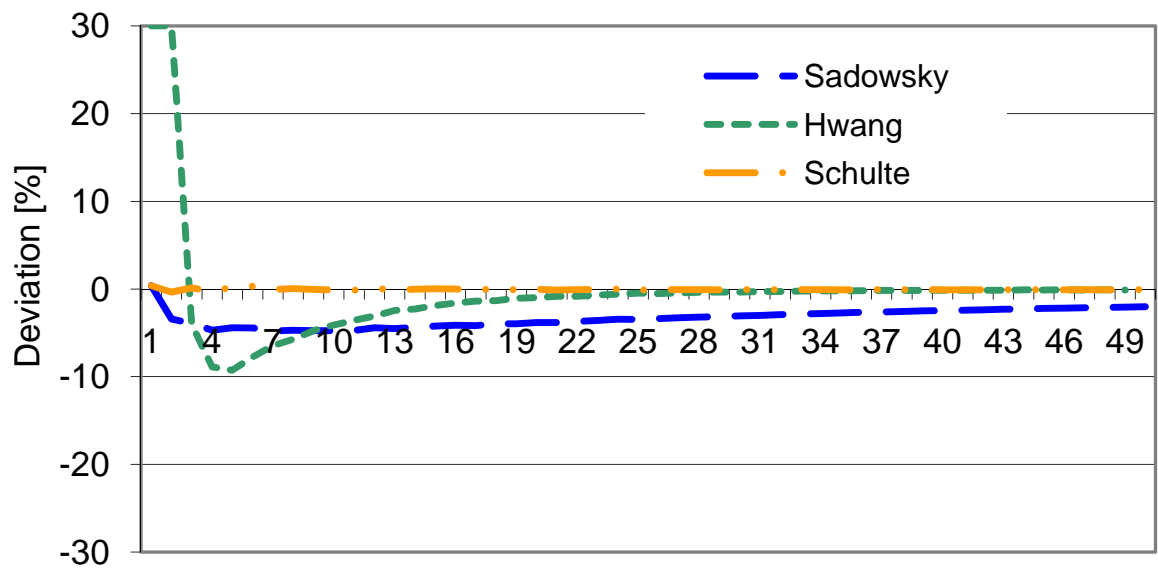

Average number of orderlines per order

Figure 17: Deviation of the calculated across-aisle distance from the simulation; System 2; uniformly distributed access frequency

In addition to Hwang's results, Schulte's calculation results must be mentioned as well. He provides a general mathematical solution for the case of a uniform distributed access rate over all storage bins [Sch96].

For all experiments, the calculation method provides values with deviations ranking between $2 \%$ and $5 \%$. The Hwang method shows a deviation of $75 \%$ for $n=1$. From $n=3$ orderlines per order and beyond, the deviation falls below 10\%. For 20 orderlines per order, the deviation is less than $1 \%$. A comparison of the simulation with Schulte's calculations shows deviations of below $1 \%$. If the number of orderlines per order increases to five, the deviation remains below $0.2 \%$.

In case that the access rate is defined by an exponential distribution, different values regarding the across-aisles distance are obtained by Hwang. For all of the other estimation methods, the values are constant. Figures 18 and 19 show the respective values for the exponential distribution: 


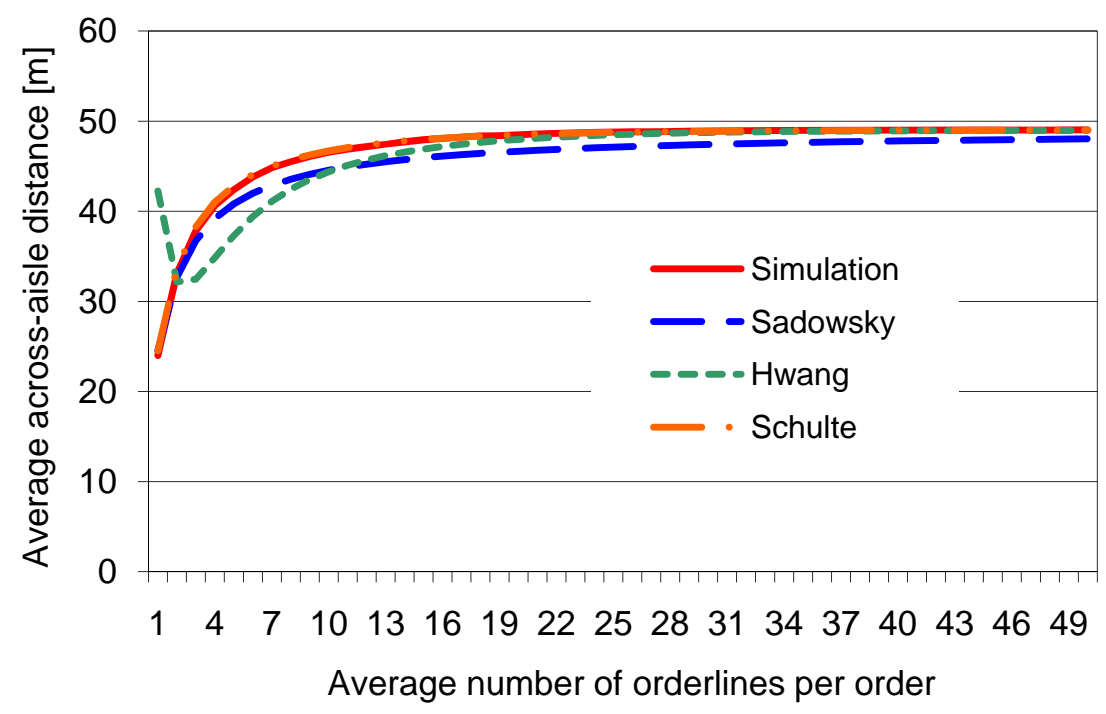

Figure 18: Across-aisle distance; System 2; exponentially distributed access frequency

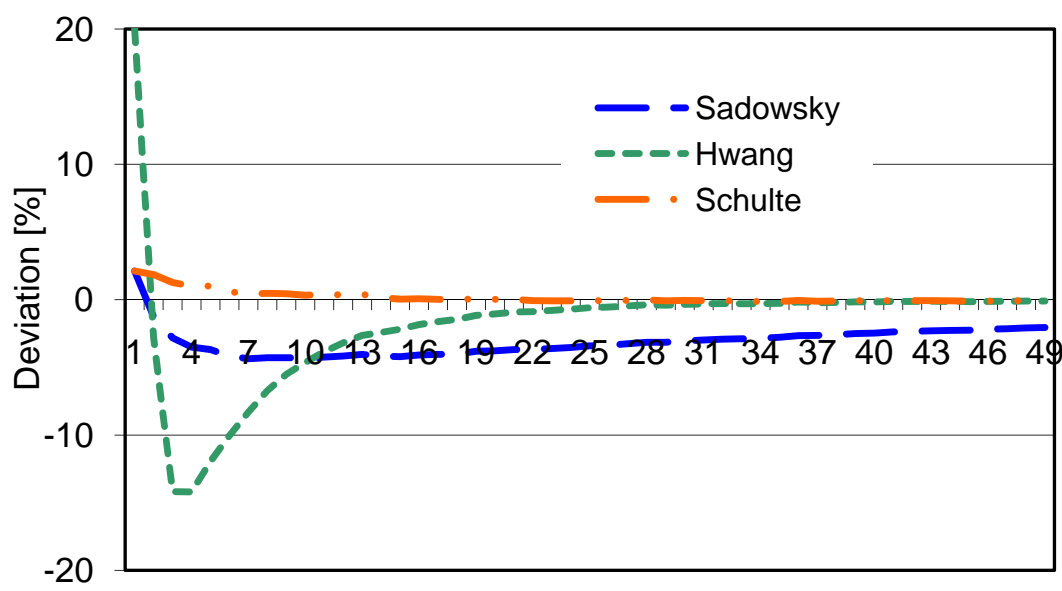

Average number of orderlines per order

Abbildung 19: Deviation of the calculated across-aisle distance from the simulation; System 2; exponentially distributed access frequency

These findings prove that the new estimation method gives the most accurate within-aisle distances for all pick path routing strategies. Even for the across-aisle distance (see Table 2), the deviations amount to $1 \%-10 \%$ depending on the strategy and the quantity of orderlines per order.

The essential findings for systems 1, 2 and 3 are pictured in Tables 2, 3 and 4.

Table 2: Number of orderlines per order $n$, for which the deviation is lower than specified; System 1; exponential distribution

\begin{tabular}{l|c|cc} 
Deviation & $10 \%$ & $5 \%$ & $1 \%$ \\
\hline $\mathrm{S}_{\mathrm{W}}-\mathrm{TSWS}$ & 6 & - & - \\
\hline $\mathrm{S}_{\mathrm{W}}-\mathrm{RSoR}$ & - & 1 & 2 \\
\hline $\mathrm{S}_{\mathrm{W}}-\mathrm{RSwR}$ & - & 1 & 2 \\
\hline $\mathrm{S}_{\mathrm{W}}-\mathrm{MH}$ & 4 & 7 & 12 \\
\hline $\mathrm{s}_{\mathrm{A}}$ & 1 & 18 & -
\end{tabular}


Table 3: Number of orderlines per order $n$, for which the deviation is lower than specified; System 2; exponential distribution

\begin{tabular}{l|c|c|c} 
Deviation & $10 \%$ & $5 \%$ & $1 \%$ \\
\hline $\mathrm{S}_{\mathrm{W}}-\mathrm{TSwS}$ & 5 & 10 & 25 \\
\hline $\mathrm{S}_{\mathrm{W}}-\mathrm{RSoR}$ & - & 1 & 3 \\
\hline $\mathrm{S}_{\mathrm{W}}-\mathrm{RSWR}$ & - & 1 & 4 \\
\hline $\mathrm{S}_{\mathrm{W}}-\mathrm{MH}$ & & 4 & 9 \\
\hline $\mathrm{S}_{\mathrm{A}}$ & - & 1 & -
\end{tabular}

Table 4: Number of orderlines per order $\mathbf{n}$, for which the deviation is lower than specified; System 3; exponential distribution

\begin{tabular}{l|c|c|c} 
Deviation & $10 \%$ & $5 \%$ & $1 \%$ \\
\hline $\mathrm{S}_{\mathrm{W}}-\mathrm{TSWS}$ & 4 & 12 & 34 \\
\hline $\mathrm{S}_{\mathrm{W}}-\mathrm{RSoR}$ & 2 & 4 & 36 \\
\hline $\mathrm{S}_{\mathrm{W}}-\mathrm{RSWR}$ & 2 & 4 & 16 \\
\hline $\mathrm{S}_{\mathrm{W}}-\mathrm{MH}$ & 6 & 12 & 33 \\
\hline $\mathrm{S}_{\mathrm{A}}$ & - & 1 & -
\end{tabular}

The deviations between calculation and simulation can mainly be attributed to discrete intervals between the bin locations (within-aisle distance) respectively between the aisles (across-aisle distance) of real picking systems. In the calculation continuous functions are used instead of discrete functions. This explains why the deviation values decrease as the bin location density increases and as the number of aisles increases.

\section{Conclusion}

The procedure for the calculation of average travel distances in low-level picker-to-part systems, as described in this paper, is a simple, applicable procedure for the planning of order picking systems. By comparison to simulated results, it has been validated that the new calculation method defined in this paper provides more precise values than current accepted methods, especially for cases with a low number of orderlines per order and storage bin assignment based on the SKU access frequencies.

For an exponentially distributed access rate within the aisle, a minimum accuracy of $90 \%$ is achieved for the Traversal Strategy with more than five orderlines per order. The precision of the calculations can be improved even further by treating the single line orders separately. For the Return Strategy With and Without Repetition, a precision of $95 \%$ is achieved with four orderlines per order or more. For the Midpoint Heuristic, accuracies of $90 \%$ can be achieved if more than five orderlines per order exists.

For the calculation of the across-aisle distance, accuracies of $93 \%$ are obtained. If the number of aisles, where order picking takes place, is increased, the accuracy of the calculation increases as well (96\% in System 3).

For the Traversal Strategy With Skips as well as for the Midpoint Heuristic, the minimum number of orderlines per order should not fall below four (Traversal Strategy) or five (Midpoint Heuristic) respectively. If the values are below these limits, the calculations have to be modified accordingly [Sad07].

Because of the accuracies achieved, the presented procedure for the determination of travel distances in lowlevel picker-to-part systems can be used to support the planning of order picking systems. The accuracy of the new method allows to analytically determine the efficiency of different combinations of layout design, storage bin assignment policies, and pick path routing strategies and to accurately select the most efficient system layout. 


\section{Literature}

[Car98]

[LeD05]

[Gen04]

[Goe88]

[Gud73]

[Gud05]

[Hal93]

[Hwa04]

[Kun75]

$[\operatorname{Mod} 73]$

[Ran90]

[Rat83]

[Roo01]

[Roo06]

[Sad07]

[Sch96]

[StH08]

[Tom03]
Caron, F; Marchet G.; Perego A.: Routing policies and COI-based bin slotting policies in picker-to-part Systems. International Journal of Production Research, 36, 1998

Le Duc, T. , de Koster, R.: Travel distance estimation and storage zone optimization in a 2-block class-based storage strategy warehouse. International Journal of Production Research, 43, 2005

Genschel, Ulrike; Becker, Claudia: Schließende Statistik. Springer Verlag, Berlin, 2004

Goetschalkx,Marc; Ratliff, H Donald, Order picking In An Aisle, IIE Transactions, 20,1988

Gudehus, Timm: Grundlagen der Kommissioniertechnik - Dynamik der Warenverteil- und Lagersysteme. Verlag W. Gidaret, Essen, 1973

Gudehus, Timm: Logistik - Grundlagen, Strategien, Anwendungen. Springer Verlag Berlin Heidelberg, 2005

Hall, Randolph W.; Distance Approximation for Routing Manual Pickers in a Warehouse. IIE Transactions, 25, 1993

Hwang, H.; Oh, Y. H.; Lee Y. K.: An evaluation of routing policies for order-picking operations in low level picker-to-part system. International Journal of Production Research, 42, 2004.

Kunder, Richard; Gudehus, Timm: Mittlere Wegzeiten beim eindimensionalen Kommissionieren. Zeitschrift für Operation Research, 1975

Modd; Graybill; Boes: Introduction to the theory of statistics. McGraw-Hill Education, 1973

Rana, Krishan, Order Picking in Narrow-aisle Warehousees, International Journal of Physical Distribution and Logistics Management, 1990

Ratliff,H. Donlad, Rosenthal, Arnon S. ; Order-Picking in a rectangular Warehouse: A solvabel Case of the Travbeling salesman Problem. Opreations Research, 31, 1993 Roodbergen,K.J.; de Koster, R.: Routing orderfillers in a warehouse with a middle aisle, European Journal of Operational Research, 133, 2001

Roodbergen,K.J.; Vis, I.F.A., A model for warehouse layout , IIE Transactions, 38, 2006

Sadowsky, Volker: Beitrag zur analytischen Leistungsermittlung von

Kommissioniersystemen, Verlag Praxiswissen, 2007

Schulte, J.: Berechnungsgrundlagen konventioneller Kommissioniersysteme.

Dissertation, 1996.

Sadowsky, Volker; ten Hompel, Michael; Calculation of the travel distance in a lowlevel picker to part system considering any distribution function within the aisles; International Material Handling Research Colloquium, Dortmund, 2008

Tompkins, J.A., White, J.A., Bozer, Y.A., Frazelle, E.H., Tanchoco, J.M.A.; Facilities Planning, 2003 


\section{Appendix}

Table 5: Within-aisle distance total and deviation from the Simulation; System 1; exponentially distributed access frequency

\begin{tabular}{|c|c|c|c|c|c|c|c|c|c|c|c|}
\hline & \multicolumn{11}{|c|}{ Within-aisle distance, total } \\
\hline & \multicolumn{3}{|c|}{ TSwS } & \multicolumn{3}{|c|}{ RSoR } & \multicolumn{2}{|c|}{ RSwS } & \multicolumn{3}{|c|}{ MH } \\
\hline$n$ & Sad & Hwa & Sim & Sad & Hwa & Sim & Sad & Sim & Sad & Hwa & Sim \\
\hline 1 & 38,0 & $\overline{0,0}$ & 10,7 & 11,0 & $\overline{1,0}$ & 10,7 & 11,0 & 10,7 & 79,9 & 73,0 & $\overline{36,9}$ \\
\hline 2 & 68,5 & 12,6 & 64,7 & 20,6 & 19,0 & 20,7 & 21,9 & 21,9 & 83,5 & 73,4 & 71,7 \\
\hline 3 & 93,0 & 19,9 & 79,4 & 29,2 & 27,3 & 29,3 & 32,9 & 33,1 & 86,9 & 77,1 & 78,8 \\
\hline 4 & 112,6 & 25,7 & 98,9 & 36,9 & 34,9 & 37,2 & 43,8 & 43,6 & 90,1 & 81,1 & 83,0 \\
\hline 5 & 128,3 & 30,8 & 116,4 & 43,8 & 41,7 & 43,5 & 54,8 & 54,6 & 93,2 & 85,2 & 87,5 \\
\hline 6 & 140,8 & 35,3 & 131,0 & 50,0 & 48,0 & 50,1 & 65,7 & 65,6 & 96,0 & 89,3 & 91,3 \\
\hline 7 & 150,9 & 39,5 & 141,8 & 55,6 & 53,7 & 55,9 & 76,7 & 76,7 & 98,6 & 93,2 & 94,7 \\
\hline 8 & 158,9 & 43,3 & 149,6 & 60,7 & 58,9 & 61,3 & 87,7 & 87,9 & 101,2 & 96,9 & 98,0 \\
\hline 9 & 165,3 & 46,7 & 154,3 & 65,3 & 63,7 & 65,9 & 98,6 & 98,5 & 103,5 & 100,5 & 101,2 \\
\hline 10 & 170,4 & 49,9 & 158,1 & 69,6 & 68,1 & 69,9 & 109,6 & 109,6 & 105,8 & 104,0 & 104,0 \\
\hline 11 & 174,4 & 52,7 & 160,7 & 73,6 & 72,1 & 74,2 & 120,5 & 121,0 & 107,9 & 107,2 & 106,6 \\
\hline 12 & 177,7 & 55,4 & 162,3 & 77,2 & 75,8 & 77,9 & 131,5 & 132,0 & 109,9 & 110,3 & 109,1 \\
\hline 13 & 180,2 & 57,7 & 164,4 & 80,6 & 79,2 & 81,4 & 142,4 & 143,0 & 111,8 & 113,3 & 111,6 \\
\hline 14 & 182,3 & 59,9 & 165,7 & 83,8 & 82,4 & 84,9 & 153,4 & 154,3 & 113,6 & 116,1 & 113,5 \\
\hline 15 & 183,9 & 61,9 & 166,8 & 86,8 & 85,4 & 88,5 & $\mid 164,4$ & 165,7 & 115,3 & 118,7 & 115,6 \\
\hline 16 & 185,2 & 63,8 & 168,0 & 89,6 & 88,1 & 90,1 & 175,3 & 176,3 & 116,9 & 121,2 & 117 \\
\hline 17 & 186,2 & 65,4 & 168,5 & 92,2 & 90,7 & 92,9 & 186,3 & 187,0 & $\mid 118,5$ & $\mid 123,5$ & 119,1 \\
\hline 18 & 187,0 & 67,0 & 169,2 & 94,7 & 93,2 & 95,1 & 197,2 & 197,7 & 120,0 & 125,7 & 120,7 \\
\hline 19 & 187,6 & 68,4 & 170,2 & 97,1 & 95,4 & 98,2 & 208,2 & 209,1 & 121,4 & 127,8 & 122,3 \\
\hline 20 & 188,1 & 69,7 & 170,9 & 99,3 & 97,6 & 100,6 & 219,1 & 220,7 & 122,7 & 129,8 & 123,7 \\
\hline 21 & 188,5 & 70,9 & 171,4 & 101,4 & 99,7 & 102,8 & 230,1 & 231,4 & 124,0 & 131,7 & 125,5 \\
\hline 22 & 188,9 & 72,0 & 171,7 & 103,5 & 101,6 & 104,4 & 241,1 & 242,4 & 125,3 & 133,4 & 126,4 \\
\hline 23 & 189,1 & 73,1 & 172,1 & 105,4 & 103,5 & 106,6 & 252,0 & 253,1 & 126,5 & 135,1 & 127,5 \\
\hline 24 & 189,3 & 74,0 & 172,3 & 107,3 & 105,3 & 108,3 & 263,0 & 264,4 & 127,6 & 136,7 & 129,1 \\
\hline 25 & 189,5 & 74,9 & 172,8 & 109,1 & 107,0 & 109,9 & 273,9 & 275,5 & 128,7 & 138,2 & 129,8 \\
\hline 26 & 189,6 & 75,7 & 173,6 & 110,8 & 108,6 & 112,6 & 284,9 & 287,4 & 129,8 & 139,6 & 131,1 \\
\hline 27 & 189,7 & 76,5 & 173,8 & 112,5 & 110,2 & 113,6 & 295,8 & 297,5 & 130,8 & 140,9 & 132,1 \\
\hline 28 & 189,7 & 77,2 & 174,0 & 114,1 & 111,7 & 114,8 & 306,8 & 307,6 & 131,8 & 142,2 & 132,8 \\
\hline 29 & 189,8 & 77,9 & 174,3 & 115,6 & 113,2 & 116,4 & 317,7 & 319,1 & 132,7 & 143,4 & 134,2 \\
\hline 30 & 189,8 & 78,5 & 174,6 & 117,1 & 114,6 & 117,9 & 328,7 & 330,1 & 133,6 & 144,6 & 134,8 \\
\hline
\end{tabular}

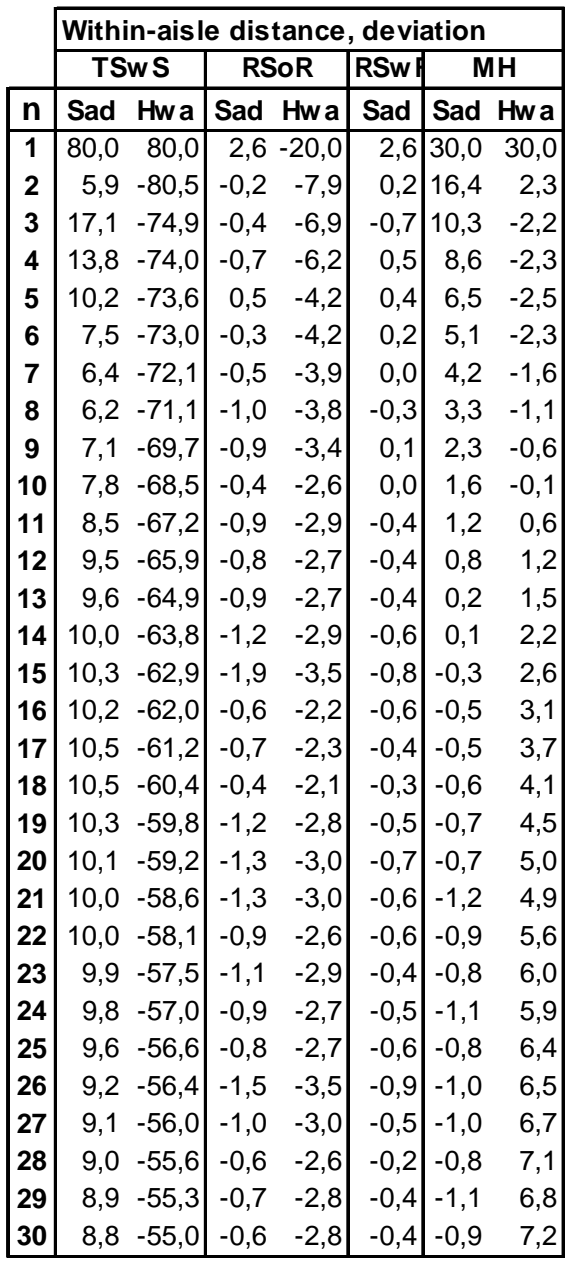

TSw S $=$ Traversal Strategy With Skips

RSoR = Return strategy Without Repetition

RSw R = Return Strategy With Repetition

$\mathrm{MH}=$ Midpoint Heuristic
Gud $=$ Gudehus

$\mathrm{Hw} \mathrm{a}=\mathrm{Hw}$ ang

Sad $=$ Sadow sky

Sch $=$ Schulte

Sim = Simulation

$\mathrm{n}=$ number of orderlines 
Tabelle 6: Across-aisle distance total and deviation from Simulation, uniform distributed access frequency over all aisles, System 1

\begin{tabular}{|c|c|c|c|c|c|c|}
\hline & \multicolumn{6}{|c|}{ Across-aisle distance, total } \\
\hline & TSw S & TSw S & TSw S & & TSw S & TSw S \\
\hline & RSoR & RSoR & & RSoR & RSoR & RSoR \\
\hline & RSw R & RSw R & & RSw R & RSw R & RSw R \\
\hline & $\mathrm{MH}$ & $\mathrm{MH}$ & & $\mathrm{MH}$ & & $\mathrm{MH}$ \\
\hline $\mathbf{n}$ & Sad & Gud & $\mathrm{Hw}$ & & Sch & Sim \\
\hline 1 & 14,00 & 14,00 & 79,72 & 21,20 & 14,00 & 13,19 \\
\hline 2 & 18,67 & 18,00 & 79,46 & 18,64 & 19,62 & 18,71 \\
\hline 3 & 21,00 & 19,86 & 79,22 & 19,57 & 22,43 & 21,67 \\
\hline 4 & 22,40 & 20,92 & 78,98 & 21,14 & 24,07 & 23,69 \\
\hline 5 & 23,33 & 21,58 & 78,76 & 22,59 & 25,12 & 24,68 \\
\hline 6 & 24,00 & 22,03 & 78,54 & 23,78 & 25,85 & 25,55 \\
\hline 7 & 24,50 & 22,35 & 78,32 & 24,72 & 26,36 & 26,06 \\
\hline 8 & 24,89 & 22,58 & 78,11 & 25,43 & 26,74 & 26,58 \\
\hline 9 & 25,20 & 22,75 & 77,89 & 25,99 & 27,02 & 26,97 \\
\hline 10 & 25,46 & 22,88 & 77,67 & 26,42 & 27,24 & 27,12 \\
\hline 11 & 25,67 & 22,97 & 77,45 & 26,75 & 27,40 & 27,34 \\
\hline 12 & 25,85 & 23,05 & 77,24 & 27,01 & 27,53 & 27,41 \\
\hline 13 & 26,00 & 23,11 & 77,02 & 27,21 & 27,63 & 27,59 \\
\hline 14 & 26,13 & 23,16 & 76,79 & 27,37 & 27,71 & 27,72 \\
\hline 15 & 26,25 & 23,19 & 76,57 & 27,50 & 27,77 & 27,77 \\
\hline 16 & 26,35 & 23,22 & 76,35 & 27,60 & 27,82 & 27,82 \\
\hline 17 & 26,44 & 23,24 & 76,12 & 27,68 & 27,86 & 27,84 \\
\hline 18 & 26,53 & 23,26 & 75,89 & 27,75 & 27,89 & 27,89 \\
\hline 19 & 26,60 & 23,28 & 75,66 & 27,80 & 27,91 & 27,92 \\
\hline 20 & 26,67 & 23,29 & 75,44 & 27,84 & 27,93 & 27,96 \\
\hline 21 & 26,73 & 23,30 & 75,21 & 27,87 & 27,95 & 27,96 \\
\hline 22 & 26,78 & 23,30 & 74,98 & 27,90 & 27,96 & 27,97 \\
\hline 23 & 26,83 & 23,31 & 74,74 & 27,92 & 27,97 & 27,98 \\
\hline 24 & 26,88 & 23,32 & 74,51 & 27,93 & 27,97 & 28,02 \\
\hline 25 & 26,92 & 23,32 & 74,28 & 27,95 & 27,98 & 28,00 \\
\hline 26 & 26,96 & 23,32 & 74,05 & 27,96 & 27,98 & 28,02 \\
\hline 27 & 27,00 & 23,32 & 73,82 & 27,97 & 27,99 & 28,02 \\
\hline 28 & 27,03 & 23,33 & 73,59 & 27,97 & 27,99 & 28,02 \\
\hline 29 & 27,07 & 23,33 & 73,36 & 27,98 & 27,99 & 28,02 \\
\hline 30 & 27,10 & 23,33 & 73,13 & 27,98 & 27,99 & 28,02 \\
\hline
\end{tabular}

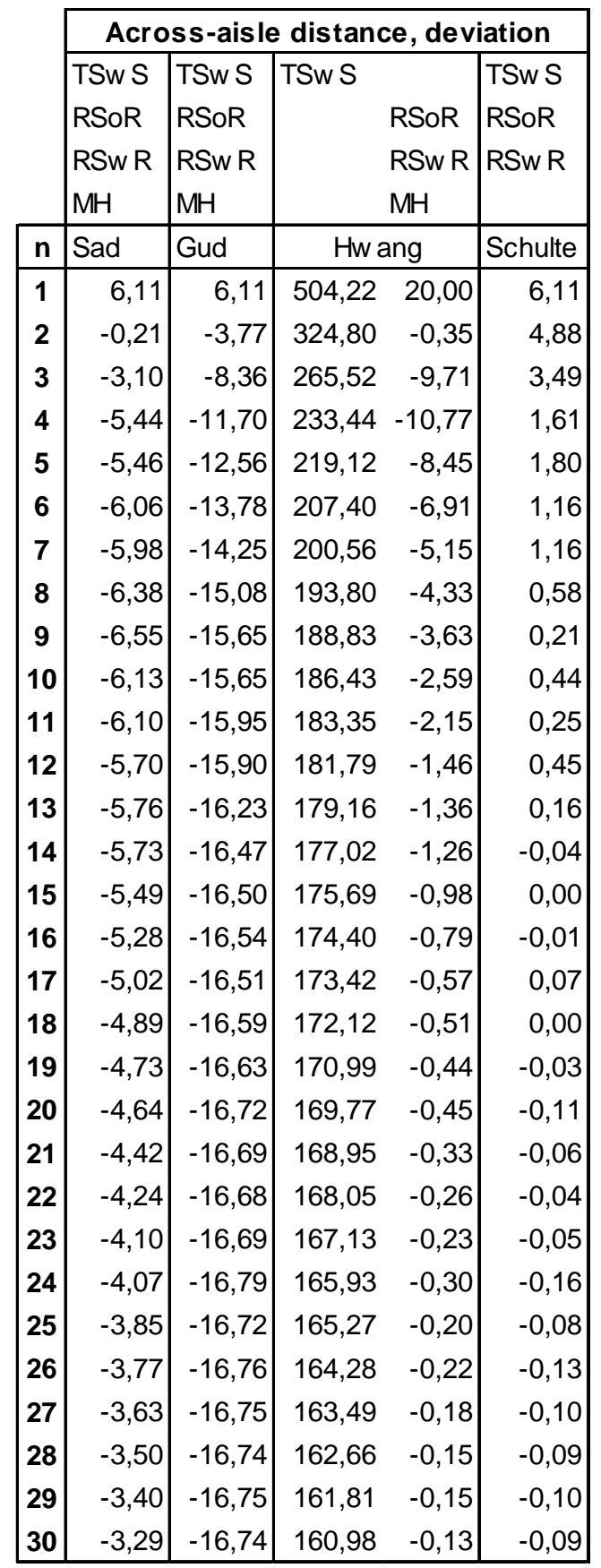


Table 7: Within-aisle distance total and deviation from Simulation, exponentially distributed access frequency; System 3

\begin{tabular}{|c|c|c|c|c|c|c|c|c|c|c|c|}
\hline & & & & & & $\theta$ & e & & & & \\
\hline & & TSw S & & & RSoR & & SI & $N \mathrm{R}$ & & $\mathrm{MH}$ & \\
\hline $\mathrm{n}$ & Sad & Hwa & Sim & Sad & $\mathrm{Hw}$ a & Sim & Sad & Sim & Sad & $\mathrm{Hw}$ a & $\mathrm{Sim}$ \\
\hline 1 & 38,0 & 37,0 & 9,7 & 11,0 & 1,0 & 9,7 & 11,0 & 9,7 & 81,2 & 73,0 & 38,1 \\
\hline 2 & 72,2 & 61,7 & 69,8 & 21,3 & 19,5 & 20,0 & 21,9 & 20,6 & 86,2 & 73,7 & 74,1 \\
\hline 3 & 103,0 & 78,8 & 83,2 & 31,0 & 28,6 & 29,4 & 32,9 & 31,2 & 91,0 & 78,3 & 80,5 \\
\hline 4 & 130,7 & 91,2 & 119,1 & 40,1 & 37,3 & 38,4 & 43,8 & 42,1 & 95,7 & 83,2 & 85,5 \\
\hline 5 & 155,7 & 100,7 & 144,0 & 48,8 & 45,7 & 47,3 & 54,8 & 52,9 & 100,3 & 88,1 & 90,9 \\
\hline 6 & 178,1 & 108,2 & 163,7 & 57,0 & 53,6 & 55,1 & 65,7 & 63,8 & 104,7 & 93,0 & 95,6 \\
\hline 7 & 198,4 & 114,6 & 186,0 & 64,7 & 61,3 & 62,9 & 76,7 & 74,8 & 109,0 & 97,8 & 100,6 \\
\hline 8 & 216,6 & 120,1 & 204,3 & 72,1 & 68,6 & 70,1 & 87,7 & 85,5 & 113,1 & 102,7 & 105,3 \\
\hline 9 & 232,9 & 124,9 & 220,2 & 79,0 & 75,5 & 77,0 & 98,6 & 96,5 & 117,1 & 107,4 & 109,4 \\
\hline 10 & 247,7 & 129,3 & 234,1 & 85,6 & 82,2 & 83,7 & 109,6 & 107,6 & 121,0 & 112,2 & 114,0 \\
\hline 11 & 261,0 & 133,4 & 248,4 & 91,9 & 88,6 & 90,2 & 120,5 & 119,0 & 124,8 & 116,9 & 118,6 \\
\hline 12 & 272,9 & 137,1 & 260,1 & 97,9 & 94,7 & 96,5 & 131,5 & 130,1 & 128,4 & 121,5 & 122,7 \\
\hline 13 & 283,6 & 140,6 & 272,3 & 103,6 & 100,5 & 102,2 & 142,4 & 140,8 & 132,0 & 126,0 & 126,8 \\
\hline 14 & 293,3 & 143,9 & 281,2 & 109,1 & 106,1 & 107,0 & 153,4 & 151,6 & 135,4 & 130,5 & 130,1 \\
\hline 15 & 302,0 & 147,0 & 291,7 & 114,3 & 111,4 & 112,5 & 164,4 & 162,4 & 138,8 & 134,9 & 134,0 \\
\hline 16 & 309,8 & 149,9 & 298,6 & 119,3 & 116,6 & 118,1 & 175,3 & 173,8 & 142,0 & 139,3 & 137,6 \\
\hline 17 & 316,9 & 152,6 & 306,0 & 124,0 & 121,5 & 122,6 & 186,3 & 184,5 & 145,2 & 143,6 & 141,1 \\
\hline 18 & 323,2 & 155,2 & 312,8 & 128,6 & 126,2 & 127,1 & 197,2 & 195,3 & 148,3 & 147,8 & 144,7 \\
\hline 19 & 328,9 & 157,7 & 318,1 & 133,0 & 130,7 & 131,5 & 208,2 & 206,5 & 151,3 & 151,9 & 147,5 \\
\hline 20 & 334,1 & 160,1 & 323,0 & 137,2 & 135,0 & 135,3 & 219,1 & 217,4 & 154,2 & 155,9 & 151,0 \\
\hline$\angle 1$ & 338,7 & 162,3 & 328,7 & 141,2 & 139,1 & 140,1 & 230,1 & 228,8 & 157,0 & 159,9 & 154,6 \\
\hline 22 & 342,8 & 164,5 & 333,4 & 145,1 & 143,1 & 143,1 & 241,1 & 238,7 & 159,8 & 163,7 & 156,7 \\
\hline 23 & 346,6 & 166,5 & 336,1 & 148,9 & 146,9 & 146,9 & 252,0 & 250,0 & 162,4 & 167,5 & 159,8 \\
\hline 24 & 349,9 & 168,5 & 341,4 & 152,5 & 150,6 & 150,8 & 263,0 & 260,9 & 165,0 & 171,2 & 162,6 \\
\hline 25 & 353,0 & 170,3 & 344,2 & 156,0 & 154,1 & 153,8 & 273,9 & 271,9 & 167,6 & 174,9 & 165,3 \\
\hline 26 & 355,7 & 172,1 & 348,6 & 159,3 & 157,5 & 157,4 & 284,9 & 282,4 & 170,1 & 178,4 & 167,5 \\
\hline 27 & 358,1 & 173,9 & 351,0 & 162,6 & 160,8 & 160,1 & 295,8 & 293,0 & 172,5 & 181,9 & 170,3 \\
\hline $2 \varepsilon$ & 360,3 & 175,5 & 354,2 & 165,7 & 164,0 & 163,1 & 306,8 & 303,7 & 174,8 & 185,2 & 172,6 \\
\hline 29 & 362,3 & 177,1 & 356,2 & 168,8 & 167,0 & 166,6 & 317,7 & 314,7 & 177,1 & 188,5 & 174,9 \\
\hline 3 & 364,1 & 178,6 & 358,1 & 171,7 & 170,0 & 169,6 & 328,7 & 325,4 & 179,4 & 191,8 & 177,4 \\
\hline 31 & 365,7 & 180,1 & 361,0 & 174,6 & 172,8 & 172,2 & 339,7 & 336,3 & 181,6 & 194,9 & 179,6 \\
\hline 3 & 367,2 & 181,5 & 363,1 & 177,4 & 175,6 & 174,8 & 350,6 & 347,3 & 183,7 & 198,0 & 181,9 \\
\hline 33 & 368,4 & 182,9 & 364,3 & 180,1 & 178,3 & 178,6 & 361,6 & 358,8 & 185,8 & 200,9 & 184,6 \\
\hline 34 & 369,6 & 184,3 & 366,0 & 182,7 & 180,9 & 180,6 & 372,5 & 369,4 & 187,8 & 203,9 & 186,2 \\
\hline 35 & 370,7 & 185,6 & 366,7 & 185,3 & 183,4 & 182,5 & 383,5 & 380,6 & 189,8 & 206,7 & 188,3 \\
\hline 3 & 371,6 & 186,8 & 369,1 & 187,7 & 185,8 & 186,2 & 394,4 & 391,8 & 191,8 & 209,5 & 190,3 \\
\hline 37 & 372,5 & 188,0 & 369,8 & 190,2 & 188,2 & 188,5 & 405,4 & 402,6 & 193,7 & 212,2 & 193,2 \\
\hline 38 & 373,2 & 189,2 & 370,6 & 192,5 & 190,5 & 190,2 & 416,4 & 413,4 & 195,5 & 214,8 & 194,1 \\
\hline 39 & 373,9 & 190,3 & 371,5 & 194,8 & 192,7 & 193,2 & 427,3 & 425,1 & 197,3 & 217,4 & 196,3 \\
\hline 40 & 374,5 & 191,5 & 372,7 & 197,0 & 194,9 & 195,4 & 438,3 & 435,8 & 199,1 & 219,8 & 198,1 \\
\hline 41 & 375,1 & 192,5 & 373,7 & 199,2 & 197,0 & 198,1 & 449,2 & 447,3 & 200,9 & 222,3 & 199,9 \\
\hline 42 & 375,6 & 193,6 & 374,3 & 201,4 & 199,0 & 199,6 & 460,2 & 458,2 & 202,6 & 224,7 & 202,2 \\
\hline 43 & 376,0 & 194,6 & 374,6 & 203,4 & 201,0 & 202,0 & 471,1 & 469,0 & 204,2 & 227,0 & 203,9 \\
\hline 4 & 376,4 & 195,7 & 375,3 & 205,5 & 203,0 & 203,4 & 482,1 & 479,2 & 205,9 & 229,2 & 205,2 \\
\hline 4 & 376,8 & 196,6 & 376,6 & 207,5 & 204,9 & 205,8 & 493,1 & 490,8 & 207,5 & 231,4 & 206,7 \\
\hline 46 & 377,1 & 197,6 & 376,3 & 209,4 & 206,8 & 207,8 & 504,0 & 501,6 & 209,0 & 233,6 & 207,9 \\
\hline 4 & 377,4 & 198,5 & 376,6 & 211,3 & 208,6 & 210,0 & 515,0 & 513,1 & 210,6 & 235,6 & 210,2 \\
\hline 48 & 377,7 & 199,5 & 376,6 & 213,2 & 210,4 & 212,0 & 525,9 & 524,1 & 212,1 & 237,7 & 211,8 \\
\hline 49 & 377,9 & 200,4 & 377,5 & 215,0 & 212,1 & 213,3 & 536,9 & 534,7 & 213,6 & 239,7 & 213,1 \\
\hline 50 & 378,1 & 201,3 & 377,7 & 216,8 & 213,8 & 215,8 & 547,8 & 546,5 & 215,0 & 241,6 & 215,0 \\
\hline
\end{tabular}

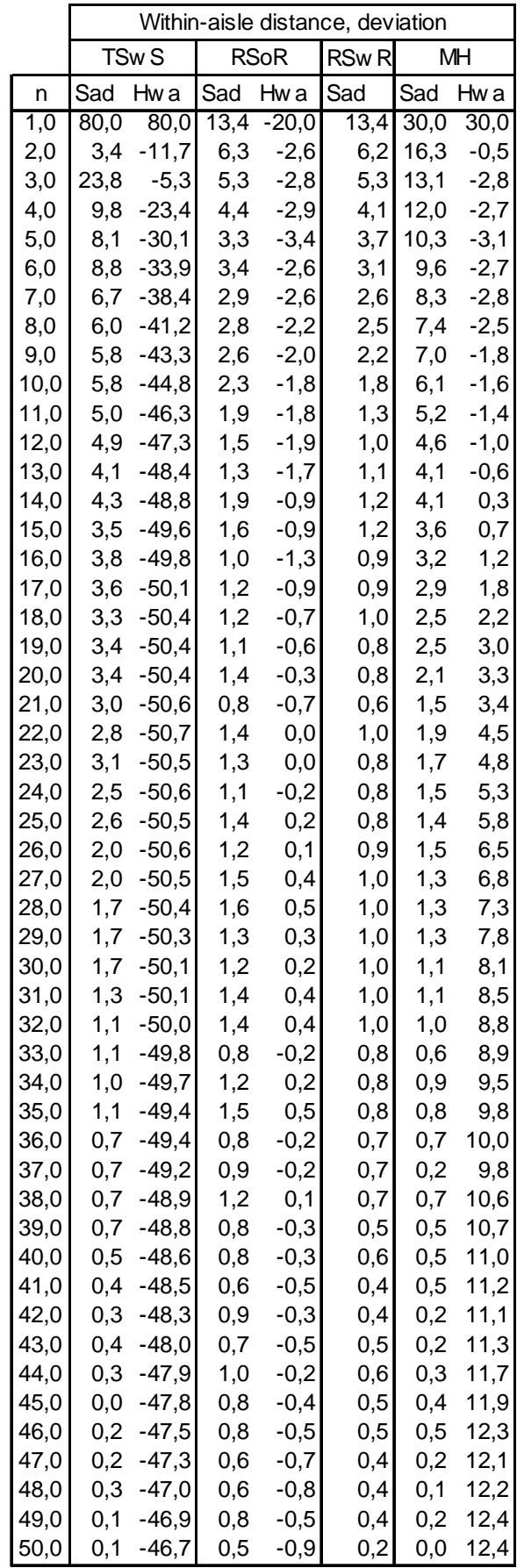


Table 8: Across-aisle distance total and deviation from Simulation, uniform distributed access frequency over all aisles, System 3

\begin{tabular}{|c|c|c|c|c|c|c|}
\hline & \multicolumn{6}{|c|}{ Across-aisle distance, total } \\
\hline & Sad & Gud & $\mathrm{HW}$ & & Sch & Sim \\
\hline & TSwS & TSWS & TSWS & & TSWS & TSwS \\
\hline & RSoR & RSoR & & SoR & RSoR & RSoR \\
\hline & RSWR & RSwR & & SWR & RSWR & RSWR \\
\hline $\mathrm{n}$ & $\mathrm{MH}$ & $\mathrm{MH}$ & & $\mathrm{H}$ & & $\mathrm{MH}$ \\
\hline 1 & 31,5 & 31,5 & 822,8 & 64,5 & 31,5 & 31,4 \\
\hline 2 & 42,0 & 41,3 & 798,5 & 44,6 & 43,1 & 42,8 \\
\hline 3 & 47,3 & 46,0 & 776,1 & 42,4 & 48,8 & 49,0 \\
\hline 4 & 50,4 & 48,8 & 755,4 & 44,5 & 52,3 & 52,2 \\
\hline 5 & 52,5 & 50,6 & 736,5 & 47,2 & 54,5 & 54,3 \\
\hline 6 & 54,0 & 51,9 & 719,0 & 49,8 & 56,2 & 56,2 \\
\hline 7 & 55,1 & 52,9 & 702,9 & 51,9 & 57,4 & 57,2 \\
\hline 8 & 56,0 & 53,6 & 688,0 & 53,6 & 58,3 & 58,1 \\
\hline 9 & 56,7 & 54,2 & 674,2 & 55,0 & 59,0 & 58,9 \\
\hline 10 & 57,3 & 54,6 & 661,4 & 56,1 & 59,6 & 59,5 \\
\hline 11 & 57,8 & 55,0 & 649,5 & 57,1 & 60,0 & 59,9 \\
\hline 12 & 58,2 & 55,3 & 638,4 & 57,9 & 60,4 & 60,4 \\
\hline 13 & 58,5 & 55,6 & 628,1 & 58,5 & 60,8 & 60,8 \\
\hline 14 & 58,8 & 55,8 & 618,4 & 59,1 & 61,0 & 61,0 \\
\hline 15 & 59,1 & 56,0 & 609,2 & 59,6 & 61,3 & 61,3 \\
\hline 16 & 59,3 & 56,1 & 600,7 & 60,0 & 61,5 & 61,5 \\
\hline 17 & 59,5 & 56,2 & 592,6 & 60,3 & 61,7 & 61,7 \\
\hline 18 & 59,7 & 56,4 & 584,9 & 60,6 & 61,8 & 61,9 \\
\hline 19 & 59,9 & 56,5 & 577,7 & 60,9 & 62,0 & 62,0 \\
\hline 20 & 60,0 & 56,6 & 570,8 & 61,1 & 62,1 & 62,1 \\
\hline 21 & 60,1 & 56,6 & 564,3 & 61,3 & 62,2 & 62,3 \\
\hline 22 & 60,3 & 56,7 & 558,0 & 61,5 & 62,3 & 62,3 \\
\hline 23 & 60,4 & 56,8 & 552,1 & 61,7 & 62,3 & 62,4 \\
\hline 24 & 60,5 & 56,8 & 546,4 & 61,8 & 62,4 & 62,5 \\
\hline 25 & 60,6 & 56,9 & 541,0 & 61,9 & 62,5 & 62,5 \\
\hline 26 & 60,7 & 56,9 & 535,7 & 62,1 & 62,5 & 62,6 \\
\hline 27 & 60,8 & 57,0 & 530,7 & 62,2 & 62,6 & 62,6 \\
\hline 28 & 60,8 & 57,0 & 525,9 & 62,2 & 62,6 & 62,7 \\
\hline 29 & 60,9 & 57,0 & 521,2 & 62,3 & 62,7 & 62,7 \\
\hline 30 & 61,0 & 57,0 & 516,7 & 62,4 & 62,7 & 62,8 \\
\hline 31 & 61,0 & 57,1 & 512,4 & 62,5 & 62,7 & 62,8 \\
\hline 32 & 61,1 & 57,1 & 508,1 & 62,5 & 62,8 & 62,8 \\
\hline 33 & 61,1 & 57,1 & 504,1 & 62,6 & 62,8 & 62,8 \\
\hline 34 & 61,2 & 57,1 & 500,1 & 62,6 & 62,8 & 62,8 \\
\hline 35 & 61,3 & 57,1 & 496,3 & 62,6 & 62,8 & 62,8 \\
\hline 36 & 61,3 & 57,2 & 492,5 & 62,7 & 62,8 & 62,9 \\
\hline 37 & 61,3 & 57,2 & 488,9 & 62,7 & 62,9 & 62,9 \\
\hline 38 & 61,4 & 57,2 & 485,3 & 62,7 & 62,9 & 62,9 \\
\hline 39 & 61,4 & 57,2 & 481,9 & 62,8 & 62,9 & 62,9 \\
\hline 40 & 61,5 & 57,2 & 478,5 & 62,8 & 62,9 & 62,9 \\
\hline 41 & 61,5 & 57,2 & 475,2 & 62,8 & 62,9 & 63,0 \\
\hline 42 & 61,5 & 57,2 & 472,0 & 62,8 & 62,9 & 63,0 \\
\hline 43 & 61,6 & 57,2 & 468,8 & 62,8 & 62,9 & 63,0 \\
\hline 44 & 61,6 & 57,2 & 465,8 & 62,9 & 62,9 & 63,0 \\
\hline 45 & 61,6 & 57,2 & 462,7 & 62,9 & 62,9 & 63,0 \\
\hline 46 & 61,7 & 57,2 & 459,8 & 62,9 & 62,9 & 63,0 \\
\hline 47 & 61,7 & 57,2 & 456,9 & 62,9 & 63,0 & 63,0 \\
\hline 48 & 61,7 & 57,2 & 454,1 & 62,9 & 63,0 & 63,0 \\
\hline 49 & 61,7 & 57,2 & 451,3 & 62,9 & 63,0 & 63,0 \\
\hline 50 & 61,8 & 57,2 & 448,6 & 62,9 & 63,0 & 63,0 \\
\hline
\end{tabular}

\begin{tabular}{|c|c|c|c|c|c|}
\hline & \multicolumn{5}{|c|}{ Across-aisle distance, deviation } \\
\hline & Sad & Gud & $\mathrm{HW}$ & & Sch \\
\hline & TSwS & TSWS & TSwS & & TSwS \\
\hline & RSoR & RSoR & & RSoR & RSoR \\
\hline & RSwR & RSWR & & RSWR & RSwR \\
\hline$n$ & $\mathrm{MH}$ & $\mathrm{MH}$ & & $\mathrm{MH}$ & \\
\hline 1 & 0,4 & 0,4 & 2523,4 & 20,0 & 0,4 \\
\hline 2 & $-2,0$ & $-3,6$ & 1764,0 & 4,0 & 0,5 \\
\hline 3 & $-3,6$ & $-6,2$ & 1482,6 & $-13,6$ & $-0,4$ \\
\hline 4 & $-3,5$ & $-6,5$ & 1347,0 & $-14,8$ & 0,1 \\
\hline 5 & $-3,4$ & $-6,8$ & 1255,4 & $-13,1$ & 0,4 \\
\hline 6 & $-3,9$ & $-7,6$ & 1179,8 & $-11,4$ & 0,0 \\
\hline 7 & $-3,7$ & $-7,7$ & 1127,8 & $-9,4$ & 0,2 \\
\hline 8 & $-3,6$ & $-7,7$ & 1084,6 & $-7,7$ & 0,3 \\
\hline 9 & $-3,7$ & $-8,0$ & 1045,1 & $-6,6$ & 0,2 \\
\hline 10 & $-3,7$ & $-8,2$ & 1011,7 & $-5,7$ & 0,1 \\
\hline 11 & $-3,6$ & $-8,2$ & 984,0 & $-4,7$ & 0,2 \\
\hline 12 & $-3,7$ & $-8,4$ & 957,4 & $-4,2$ & 0,1 \\
\hline 13 & $-3,8$ & $-8,6$ & 933,3 & $-3,7$ & 0,0 \\
\hline 14 & $-3,6$ & $-8,6$ & 913,4 & $-3,2$ & 0,0 \\
\hline 15 & $-3,7$ & $-8,7$ & 893,6 & $-2,9$ & $-0,1$ \\
\hline 16 & $-3,6$ & $-8,8$ & 876,8 & $-2,5$ & 0,0 \\
\hline 17 & $-3,6$ & $-8,8$ & 860,3 & $-2,3$ & $-0,1$ \\
\hline 18 & $-3,5$ & $-8,9$ & 845,5 & $-2,0$ & $-0,1$ \\
\hline 19 & $-3,5$ & $-9,0$ & 831,0 & $-1,9$ & $-0,2$ \\
\hline 20 & $-3,4$ & $-8,9$ & 819,2 & $-1,6$ & 0,0 \\
\hline 21 & $-3,4$ & $-9,0$ & 806,1 & $-1,5$ & $-0,2$ \\
\hline 22 & $-3,2$ & $-9,0$ & 795,9 & $-1,2$ & 0,0 \\
\hline 23 & $-3,2$ & $-9,0$ & 785,4 & $-1,1$ & 0,0 \\
\hline 24 & $-3,2$ & $-9,0$ & 774,9 & $-1,0$ & $-0,1$ \\
\hline 25 & $-3,0$ & $-9,0$ & 765,9 & $-0,9$ & 0,0 \\
\hline 26 & $-3,0$ & $-9,0$ & 756,3 & $-0,8$ & $-0,1$ \\
\hline 27 & $-3,0$ & $-9,1$ & 747,3 & $-0,8$ & $-0,1$ \\
\hline 28 & $-2,9$ & $-9,1$ & 739,2 & $-0,7$ & $-0,1$ \\
\hline 29 & $-2,9$ & $-9,1$ & 731,2 & $-0,6$ & $-0,1$ \\
\hline 30 & $-2,9$ & $-9,1$ & 723,3 & $-0,6$ & $-0,1$ \\
\hline 31 & $-2,8$ & $-9,1$ & 716,0 & $-0,5$ & $-0,1$ \\
\hline 32 & $-2,7$ & $-9,1$ & 709,0 & $-0,5$ & $-0,1$ \\
\hline 33 & $-2,7$ & $-9,1$ & 702,2 & $-0,4$ & $-0,1$ \\
\hline 34 & $-2,6$ & $-9,1$ & 695,8 & $-0,4$ & $-0,1$ \\
\hline 35 & $-2,5$ & $-9,1$ & 689,8 & $-0,3$ & 0,0 \\
\hline 36 & $-2,5$ & $-9,1$ & 683,0 & $-0,3$ & $-0,1$ \\
\hline 37 & $-2,5$ & $-9,1$ & 677,0 & $-0,3$ & $-0,1$ \\
\hline 38 & $-2,5$ & $-9,2$ & 671,1 & $-0,3$ & $-0,1$ \\
\hline 39 & $-2,4$ & $-9,1$ & 665,6 & $-0,3$ & $-0,1$ \\
\hline 40 & $-2,4$ & $-9,1$ & 660,1 & $-0,3$ & $-0,1$ \\
\hline 41 & $-2,3$ & $-9,2$ & 654,6 & $-0,3$ & $-0,1$ \\
\hline 42 & $-2,3$ & $-9,2$ & 649,4 & $-0,2$ & $-0,1$ \\
\hline 43 & $-2,2$ & $-9,1$ & 644,7 & $-0,2$ & $-0,1$ \\
\hline 44 & $-2,2$ & $-9,1$ & 639,6 & $-0,2$ & $-0,1$ \\
\hline 45 & $-2,1$ & $-9,1$ & 634,7 & $-0,2$ & $-0,1$ \\
\hline 46 & $-2,1$ & $-9,1$ & 629,9 & $-0,2$ & $-0,1$ \\
\hline 47 & $-2,1$ & $-9,1$ & 625,5 & $-0,1$ & 0,0 \\
\hline 48 & $-2,0$ & $-9,1$ & 620,9 & $-0,1$ & 0,0 \\
\hline 49 & $-2,0$ & $-9,2$ & 616,1 & $-0,2$ & $-0,1$ \\
\hline 50 & $-2,0$ & $-9,1$ & 611,9 & $-0,1$ & $-0,1$ \\
\hline
\end{tabular}

